\title{
CONSTRUCTIVE DESCRIPTION OF THE BESOV CLASSES IN CONVEX DOMAINS IN $\mathbb{C}^{d}$
}

\section{A. S. Rotkevich*}

UDC 517.5

The method of pseudoanalytic continuation developed by E. M. Dyn'kin is extended to convex domains in $\mathbb{C}^{d}$ and used to give a constructive description of the Besov classes in such domains. Bibliography: 22 titles.

\section{INTRODUCTION}

In 1981, E. M. Dyn'kin [5] gave a constructive description of the analytic Besov classes in terms of global polynomial estimates for the Radon domains in $\mathbb{C}$. In the present paper, the methods suggested in [5] are generalized to the multidimensional case.

Study of characteristics of functional spaces in terms of approximation is a classical problem issued by the remark of Jackson (1911) who stated that smoothness can be described in this way. One of the first results in this field is the Jackson-Bernstein theorem characterizing the periodic Hölder class $\Lambda^{s}[-\pi, \pi]$ for $0<s<1$ as the class of functions whose best approximations by trigonometric polynomials of degree $n$ decrease as $n^{-s}$ with growth of $n$. To mension results in the multidimensional case, we note that an analogous characteristic was achieved by N. A. Shirokov in [20] for the analytic Hölder classes in strictly preudocon domains. It is interesting that our result appears to be similar to the classical characterization of the $\left[0\right.$ ov $\left[\right.$ ses $\dot{B}_{p q}^{s}[-\pi, \pi]$.

Theorem 0.1. A function $f$ on $[-\pi, \pi]$ belongs to the Besov class $\dot{B}_{p, q}^{s}[-\pi, \pi], s>0,1 \leq p, q \leq \infty$, if and only if

$$
\left[\sum_{n=1}^{\infty} \frac{1}{n}\left(n^{s} E_{n}(f)_{p}\right)^{q}\right]^{1 / q}<\infty
$$

where $E_{n}(f)_{p}=\inf _{T_{n}}\left(\int_{-\pi}^{\pi}\left|f(x)-T_{n}(x)\right|^{p} d x\right)^{1 / p}$ is the best approximation of the function $f$ in $L^{p}[-\pi, \pi]$ by trigonometric polynomials of degree $n$

The method of studying the Bespaces is based on a generalization of pseudoanalytic continuation, i.e., continuation of a function $f$ defined in some bounded domain $\Omega \subset \mathbb{C}^{d}$ to a function $\mathbf{f}$ defined in the entire space $\mathbb{C}^{d}$ such that the discrepancy of the Cauchy-Riemann equations $|\bar{\partial} \mathbf{f}|=\left|\frac{\partial \mathbf{f}}{\partial \bar{z}_{1}}\right|+\cdots+\left|\frac{\partial \mathbf{f}}{\partial \bar{z}_{d}}\right|$ decreases in a controlled way with the approach to the boundary of the domain $\Omega$. The rate of this decreasing uniqely characterizes smoothness of the initial function. Similarly to the one-dimensional case, a function $f \in H^{p}(\Omega)$ belongs to the analytic Besov space $A_{p}^{s}(\Omega)$ if and only if its continuation with

$$
\int_{\mathbb{C}^{d} \backslash \Omega}|\bar{\partial} \mathbf{f}(z)|^{p} \rho(z)^{-p(s-1)-1} d \mu(z)<\infty
$$

is possible, where $\rho$ is the function defining the domain $\Omega$ and $\mu$ is Lebesgue measure in $\mathbb{C}^{d}$.

The main idea is that completely different constructions of pseudoanalytic continuation lead to bounds of the form (0.2). In the present paper, two constructions of continuation are introduced, based on local and globa polynomial estimations. In such a way, it becomes possible to connect the module of smoothness of a function 2 with its global estimates, which leads to statement analogous to Theorem 0.1 .

The paper is divided into seven sections. In Sec. 1, the main notation, introductional definitions, and properties of the studied functions classes are given. Section 2 is devoted to the study of the Cauchy-Leray-Fantappie formula, which is analogous to the Cauchy formula. Estimates of the kernel of this formula presented in this section are mainly obtained in [7] for a more general class of domains. Part of the estimates happens to hold only under the condition of strict convexity of the domain. As was mentioned, we study the smoothness of functions using local approximations but the classic definition of the Besov spaces is based on the module of

*St. Petersburg State University, St. Petersburg, Russia, e-mail: rotkevichas@gmail.com.

Translated from Zapiski Nauchnykh Seminarov POMI, Vol. 416, 2013, pp. 136-174. Original article submitted May 21, 2013. 
smoothness defined by the operator of taking of differences. In Sec. 3, we study the connection of the classic definition with the polynomial module of smoothness defined using local approximations. Note that the results of this section hold for an arbitrary smooth domain in $\mathbb{R}^{l}$. In Sec. 4, we construct an operator that gives "almost the best" approximation of a function by polynomials of degree not exceeding some parameter $m \geq 0$. In Sec. 5, two constructions of pseudoanalytic continuation are presented, by means of local and global polynomial approximations. In Sec. 6, the first construction is used for describing the Besov classes in terms of pseudoanalytic continuation. Particularly, it is proved that the analytic Besov classes $A_{p q}^{s}(\Omega)$ are described as the classes of functions admitting a continuation with the bound

$$
\int_{0}^{\infty}\left(\int_{\partial \Omega_{r}}|\bar{\partial} F(z)|^{p} d \sigma_{r}(z)\right)^{q / p} r^{-q(s-1)-1} d r<\infty
$$

where $\partial \Omega_{r}=\left\{z \in \mathbb{C}^{d} \backslash \Omega: \rho(z)=r\right\}, \sigma_{r}$ is the surface Lebesgue measure on $\partial \Omega_{r}$, and $\rho$ is the function defining the domain $\Omega$.

In Sec. 7, a constructive characteristic of the analytic Besov classes is studied. Particularly, it is proved that the class $A_{p q}^{s}(\Omega)$ is characterized by the following condition on the best polynomial approximations :

$$
\left[\sum_{n=1}^{\infty} \frac{1}{n}\left(n^{s} E_{n}(f)_{p}\right)^{q}\right]^{1 / q}<\infty
$$

where $E_{n}(f)_{p}=\inf _{T_{n}}\left(\int_{\partial \Omega}\left|f(z)-T_{n}(z)\right|^{p} d \sigma(z)\right)^{1 / p}$ is the best approximation of a function $f$ in $L^{p}(\partial \Omega)$ by polynomials of degree $n$ in each variable and $\sigma$ is the surface Lebesgue measure on $\partial \Omega$.

In Sec. 8, the pseudoanalytic continuation method is presented and the continuity of the Cauchy-LerayFantappiè operator in the Besov spaces $B_{p}^{s}(\partial \Omega)$ for $1<p<\infty$ and $0<s<1$ is proved.

\section{MAin DEFinitions}

1.1. Main notation. Let $\mathbb{C}^{d}$ be the space of $d$ complex variables, $d \geq 2, z=\left(z_{1}, \ldots, z_{d}\right), z_{j}=x_{j}+i y_{j}$,

$$
\begin{gathered}
\frac{\partial f}{\partial z_{j}}=\frac{1}{2}\left(\frac{\partial f}{\partial x_{j}}-i \frac{\partial f}{\partial y_{j}}\right), \quad \frac{\partial f}{\partial \bar{z}_{j}}=\frac{1}{2}\left(\frac{\partial f}{\partial x_{j}}+i \frac{\partial f}{\partial y_{j}}\right) \\
\partial f=\sum_{k=1}^{d} \frac{\partial f}{\partial z_{k}} d z_{k}, \quad \bar{\partial} f=\sum_{k=1}^{d} \frac{\partial f}{\partial \bar{z}_{k}} d \bar{z}_{k}, \quad \text { and } \quad d f=\partial f+\bar{\partial} f .
\end{gathered}
$$

Define in the space $\mathbb{C}^{d}$ the inner product $\langle z, w\rangle=\sum_{k=1}^{d} z_{k} w_{k}$ and the corresponding norm $|z|=\sqrt{\langle z, \bar{z}\rangle}$. We similarly denote the action of the differential forms $\partial f$ and $\bar{\partial} f$ on a vector $w \in \mathbb{C}^{d}$ :

$$
\langle\partial f, w\rangle=\sum_{k=1}^{d} \frac{\partial f}{\partial z_{k}} w_{k}, \quad\langle\bar{\partial} f, w\rangle=\sum_{k=1}^{d} \frac{\partial f}{\partial \bar{z}_{k}} \bar{w}_{k}
$$

Considering this notation, we usually identify the form $\partial f$ with the corresponding vector $\left(\frac{\partial f}{\partial z_{1}}, \ldots, \frac{\partial f}{\partial z_{d}}\right)$. Note that if the function $\rho$ is real-valued, then $\frac{\partial \rho}{\partial z_{k}}=\overline{\frac{\partial \rho}{\partial \bar{z}_{k}}}$.

Denote the distance from a point $z \in \mathbb{C}^{d}$ to a set $D \subset \mathbb{C}^{d}$ by $\operatorname{dist}(z, D)=\inf \{|z-w|: w \in D\}$.

To shorten the notation in inequalities, we define the symbols $\lesssim$ and $\asymp$ in the following way: $f \lesssim g$ if $f \leq c g$ for some constant $c>0$, independent of the main arguments of $f$ and $g$. Similarly, $f \asymp g$ if $c^{-1} g \leq f \leq c g$ for some constant $c>1$.

Denote by $\mu_{l}$ the $l$-dimensional Lebesgue measure. 


$$
\Omega=\left\{z \in \mathbb{C}^{d}: \rho(z)<0\right\}
$$

be a strictly convex domain such that $\partial \rho \neq 0$ on $\partial \Omega$, where $\rho$ is a function of class $C^{\infty}$. Note that we can take $\rho(z)>0$ outside the domain $\Omega$, and domains of the form $\Omega_{r}=\left\{z \in \mathbb{C}^{d}: \rho(z)<r\right\}$ are also strictly convex for $0 \leq r \leq 2$ and $\partial \rho(z) \neq 0, z \in \Omega_{2} \backslash \Omega$. This means that the second differential of the function $\rho$ generates a strictly positive definite quadratic form on the tangent plane:

$$
d^{2} \rho(z)[z-w] \geq c|z-w|^{2}, \quad \operatorname{Re}\langle\partial \rho(z), z-w\rangle=0, \quad z \in \Omega_{2} \backslash \Omega
$$

for some constant $c>0$, where $d^{2} \rho$ is the second differential of the function $\rho$. Since the function $\rho$ is real-valued,

$$
d^{2} \rho(z)[v]=2 \operatorname{Re} \sum_{k, j=1}^{d}\left(\frac{\partial^{2} \rho(z)}{\partial z_{k} \partial z_{j}} v_{k} v_{j}+\frac{1}{2} \frac{\partial^{2} \rho(z)}{\partial z_{k} \partial \bar{z}_{j}} v_{k} \bar{v}_{j}\right), \quad v \in \mathbb{C}^{d} .
$$

Consider at a point $\xi \in \partial \Omega_{r}=\left\{\xi \in \mathbb{C}^{d}: \rho(\xi)=r\right\}$ the tangent hyperplane

$$
T_{\xi}^{\mathbb{R}}=\left\{z \in \mathbb{C}^{d}: \operatorname{Re}\langle\partial \rho(\xi), \xi-z\rangle=0\right\} .
$$

This $(2 d-1)$-dimensional real affine subspace $\mathbb{C}^{d}$ contains the unique complex affine subspace $T_{\xi}$ of dimension $d-1$, which is called the complex tangent hyperplane. In our notation,

$$
T_{\xi}=\left\{z \in \mathbb{C}^{d}:\langle\partial \rho(\xi), \xi-z\rangle=0\right\} .
$$

Denote the projection of a point $z \in \mathbb{C}^{d}$ to a tangent hyperplane $T_{\xi}^{\mathbb{R}}$ by $\operatorname{pr}_{\xi}(z) \in T_{\xi}^{\mathbb{R}}$ and denote the projection to a complex tangent hyperplane by $\pi_{\xi}(z) \in T_{\xi}$.

1.3. Hardy and Besov spaces. The main objects of the following study are the Besov classes and their analytic analogs in convex domains. To define these spaces, it is necessary to iptnduce the notion of integral modulus of smoothness and the corresponding operators of taking the differences $\$$ te that the definition given in this section is inconvenient to be applied to the study of polynomial approximations. In Sec. 3, we give an equivalent definition of the Besov class in terms of best local polynomial approximations.

Thus, the spaces $L^{p}\left(\partial \Omega_{r}\right)=L^{p}\left(\partial \Omega_{r}, d \sigma_{r}\right)$ are defined in terms of the Lebesgue measure $d \sigma_{r}$ on the surface $\partial \Omega_{r}$. Denote the space of functions that are analytic in a domain $\Omega$ by $H(\Omega)$ and denote the Hardy space in the following way:

$$
H^{p}(\Omega)=\left\{f \in H(\Omega):\|f\|_{H^{p}(\Omega)}=\sup _{r<0}\|f\|_{L^{p}\left(\partial \Omega_{r}\right)}<\infty\right\}
$$

Recall that a function $f \in H^{p}(\Omega)$ takes nontangent boundary values almost everywhere. Continuing the function to the boundary, we have $\|f\|_{H^{p}(\Omega)} \asymp\|f\|_{L^{p}(\partial \Omega)}$.

Let $f \in L^{p}\left(\mathbb{R}^{l}\right), k \in \mathbb{N}$, and $t>0$, and define the operator of taking the $k$ th difference $\Omega$, $\Omega$ ordinate $e_{j}=(0, \ldots, 1, \ldots, 0)$ :

$$
\Delta_{j, t}^{1} f(x)=f\left(x+t e_{j}\right)-f(x), \quad \Delta_{j, t}^{k} f(x)=\Delta_{j, t}^{1}\left(\Delta_{j, t}^{k-1} f\right)(x) .
$$

Let $\alpha=\left(\alpha_{1}, \ldots, \alpha_{l}\right)$ be a multiindex and let $h \in \mathbb{R}^{l}$; we set

$$
\Delta_{h}^{\alpha} f(x)=\Delta_{1, h_{1}}^{\alpha_{1}} \circ \ldots \circ \Delta_{l, h_{l}}^{\alpha_{l}} f(x) .
$$

Define the corresponding $\alpha$-modulus of smoothness:

$$
\omega^{\alpha}(f, h)_{p}=\left\|\Delta_{h}^{\alpha} f\right\|_{L^{p}\left(\mathbb{R}^{l}\right)}=\left(\int_{\mathbb{R}^{l}}\left|\Delta_{h}^{\alpha} f(x)\right|^{p} d x\right)^{1 / p} .
$$

In what follows, usually $\alpha=(m, \ldots, m)$ and $h=(t, \ldots, t)$; in this case, we use a shorter notation:

$$
\omega^{m}(f, t)=\omega^{(m, \ldots, m)}(f,(t, \ldots, t)) .
$$

Definition 1.1. Let $0<s<\infty$ and $1 \leq p, q \leq \infty$. Then the Besov class $B_{p q}^{s}\left(\mathbb{R}^{l}\right)$ consists of all functions such that for $1 \leq q<\infty$, the inequality

$$
c_{p q}(f)=\left(\int_{0}^{\infty}\left(\frac{\omega^{\alpha}(f, h)_{p}}{h^{s}}\right)^{q} \frac{d h}{h}\right)^{\frac{1}{q}}<\infty
$$


holds, and for $q=\infty$, the inequality

$$
c_{p \infty}(f)=\sup _{h>0} \frac{\omega^{\alpha}(f, h)_{p}}{h^{s}}<\infty
$$

holds, where $\alpha$ is an arbitrary multiindex satisfying the condition $\alpha, i=1, \ldots, l$. The definition does not depend on the multiindex $\alpha$, and the norm $\|f\|_{B_{p q}^{s}\left(\mathbb{R}^{l}\right)}=\|f\|_{L^{p}\left(\mathbb{R}^{l}\right)}+c_{p q}(f)$ defines on $B_{p q}^{s}\left(\mathbb{R}^{l}\right)$ the structure of a Banach space. The space $B_{p p}\left(\mathbb{R}^{l}\right)$ we shortly denote by $B_{p}\left(\mathbb{R}^{l}\right)$.

Now we transfer the definition of the Besov class to the boundary of the domain $\Omega$. For this purpose, consider an open atlas and the corresponding partition of unity. Let $\partial \Omega=\bigcup_{j=1}^{N} K_{j}$ and smooth diffeomorphisms $\psi_{j}: \bar{K}_{j} \rightarrow Q_{1}$ be given, where $Q_{1}=[0,1]^{2 d-1}$. In addition, a smooth partition of unity $\sum_{j=1}^{N} \chi_{j}(z)=1, z \in \partial \Omega$, is given such that supp $\chi_{j} \subset K_{j}$. Denote the diffeomorphism that is inverse to $\psi_{j}$ by $\varphi_{j}=\psi_{j}^{-1}$.

Definition 1.2. Let $0<s<\infty$ and $1 \leq p, q \leq \infty$. Then

$$
B_{p q}^{s}(\partial \Omega)=\left\{f \in L^{p}(\partial \Omega):\left(\chi_{j} f\right) \circ \psi_{j}^{-1} \in B_{p q}^{s}\left(\mathbb{R}^{2 d-1}\right)\right\},
$$

and this space is a Banach space with the norm

$$
\|f\|_{B_{p q}^{s}(\partial \Omega)}=\sum_{j=1}^{N}\left\|\left(\chi_{j} f\right) \circ \psi_{j}^{-1}\right\|_{B_{p q}^{s}\left(\mathbb{R}^{2 d-1}\right)} .
$$

Note that different classes and partitions of unity generate different but pairly equivalent norms. To study the Besov spaces and corresponding concepts and methods in more detail, see the monograph [22].

The main object of our study is the analytic Besov class $A_{p q}^{s}(\Omega)$ consisting of all analytic functions with boundary values in the class $B_{p q}^{s}(\partial \Omega)$, and the norm in the space $A_{p q}^{s}(\Omega)$ is defined by the relation $\|f\|_{A_{p q}^{s}(\Omega)}=$ $\|f\|_{B_{p q}^{s}(\partial \Omega)}$. Respectively, $A_{p}^{s}(\Omega)=A_{p p}^{s}(\Omega)$.

1.4. The Hardy inequality. To prove some estimates, we need the following Hardy inequality. Let a function $f(t)$ be positive on $(0, \infty)$. Define a function $F(x)$ with respect to a parameter $r \neq 1$ in the following way:

$$
F(x)=\int_{0}^{x} f(t) d t, \quad r>1
$$

and

$$
F(x)=\int_{x}^{\infty} f(t) d t, \quad r<1
$$

Then for $1 \leq p<\infty$, the estimate

$$
\int_{0}^{\infty} x^{-r} F^{p}(x) d x<\left(\frac{p}{|r-1|}\right)^{p} \int_{0}^{\infty} x^{-r}(x f(x))^{p} d x
$$

holds. A proof and detailed discussion of these inequalities can be found in the monograph [8].

\section{The Cauchy-Leray-Fantappiè Formula And its Kernel estimates}

2.1. Preliminary remarks. In the theory of functions of several complex variables, an analog of the Cauchy formula is the famous Leray theorem $([1,15])$. Recall that if a domain $\Omega=\left\{z \in \mathbb{C}^{d}: \rho(z)<0\right\}$ is convex and a function $\rho$ is smooth, then a function $f \in H^{1}(\Omega)$ can be reconstructed by its boundary values using the Cauchy-Leray-Fantappiè formula:

$$
f(z)=K_{d} f(z)=\frac{1}{(2 \pi i)^{d}} \int_{\partial \Omega} \frac{f(\xi) \partial \rho(\xi) \wedge(\bar{\partial} \partial \rho(\xi))^{d-1}}{\langle\partial \rho(\xi), \xi-z\rangle^{d}}, \quad z \in \Omega .
$$

Note that according to [7], the operator $K_{d}$ continuously maps the space $L^{p}(\partial \Omega)$ onto the space $H^{p}(\Omega)$, i.e., $\left\|K_{d} f\right\|_{H^{p}(\Omega)} \lesssim\|f\|_{L^{p}(\partial \Omega)}$. 
The main instrument in this paper is the method of continuation of a function $f$ outside the domain $\Omega$. Let $f_{0} \in H^{1}(\Omega)$ and let the boundary values of the function $f_{0}$ almost everywhere concide with the boundary values of some function $F \in C_{\text {loc }}^{1}\left(\mathbb{C}^{d} \backslash \Omega\right)$ such that $|\bar{\partial} F| \in L^{1}\left(\mathbb{C}^{d} \backslash \Omega\right)$. Then by the Stokes formula, for $z \in \partial \Omega$, we have the equalities

$$
\begin{aligned}
f_{0}(z) & =\lim _{r \rightarrow 0+} \frac{1}{(2 \pi i)^{d}} \int_{\partial \Omega_{r}} \frac{F(\xi) \partial \rho(\xi) \wedge(\bar{\partial} \partial \rho(\xi))^{d-1}}{\langle\partial \rho(\xi), \xi-z\rangle^{d}} \\
& =\lim _{r \rightarrow 0+} \frac{1}{(2 \pi i)^{d}} \int_{\mathbb{C}^{d} \backslash \Omega_{r}} \frac{\bar{\partial} F(\xi) \wedge \partial \rho(\xi) \wedge(\bar{\partial} \partial \rho(\xi))^{d-1}}{\langle\partial \rho(\xi), \xi-z\rangle^{d}} \\
& =\frac{1}{(2 \pi i)^{d}} \int_{\mathbb{C}^{d} \backslash \Omega} \frac{\bar{\partial} F(\xi) \wedge \partial \rho(\xi) \wedge(\bar{\partial} \partial \rho(\xi))^{d-1}}{\langle\partial \rho(\xi), \xi-z\rangle^{d}}
\end{aligned}
$$

since

$$
d\left(\frac{\partial \rho(\xi) \wedge(\bar{\partial} \partial \rho(\xi))^{d-1}}{\langle\partial \rho(\xi), \xi-z\rangle^{d}}\right)=0, \quad z \in \Omega, \quad \xi \in \mathbb{C}^{d} \backslash \Omega
$$

This remarkable formula allows one to study properties of the function $f_{0}$ relying on estimates of its continuation. Note that it is not necessary for the function $F$ to be a continuation in terms of coincidence of boundary values; in fact, relation $(2.2)$ is sufficient. We call such a function $F$ the pseudoanalytic continuation of the function $f_{0}$.

To shorten the notation, denote

$$
\omega(\xi)=\frac{1}{(2 \pi i)^{d}} \partial \rho(\xi) \wedge(\bar{\partial} \partial \rho(\xi))^{d-1} \quad \text { and } \quad K(\xi, z)=\frac{1}{\langle\partial \rho(\xi), \xi-z\rangle^{d}} .
$$

2.2. Pointwise estimates of the kernel. As was mentioned before, an important instrument in studying boundary properties of analytic functions is formula (2.2). In this section, we give pointwise and integral estimates of the kernel of the operator $K_{d}$. Note that a part of this estimates can be obtained using only the strict linear convexity (see [7]); this refers to estimates on the surface $\partial \Omega$; however, continuation of these estimates to the set $\mathbb{C}^{d} \backslash \Omega$ essentially uses the convexity of the domain (see Lemma 2.1). For short, denote $v(\xi, z)=|\langle\partial \rho(\xi), \xi-z\rangle|$ and denote by $\xi^{*}$ a point of the boundary $\partial \Omega$ that is the closest one to a point $\xi \in \mathbb{C}^{d} \backslash \Omega$.

Lemma 2.1. Let a domain $\Omega$ be convex. Then

$$
v(\xi, z) \asymp \rho(\xi)+v\left(\xi^{*}, z\right), \quad z \in \Omega, \quad \xi \in \mathbb{C}^{d} \backslash \Omega .
$$

Proof. Denote by $n(\xi)=\frac{\partial \rho(\xi)}{|\partial \rho(\xi)|}$ the complex normal at the point $\xi$. Note that

$$
\left\langle n(\xi), \xi-\xi^{*}\right\rangle=\left|\xi-\xi^{*}\right|=\operatorname{dist}(\xi, \partial \Omega) \asymp \rho(\xi) ;
$$

hence,

$$
\begin{aligned}
\langle\partial \rho(\xi), \xi-z\rangle & =|\partial \rho(\xi)|\left(\left\langle n(\xi), \xi-\xi^{*}\right\rangle+\left\langle n(\xi), \xi^{*}-z\right\rangle\right) \\
& =|\partial \rho(\xi)|\left(\operatorname{dist}(\xi, \partial \Omega)+\left\langle n\left(\xi^{*}\right), \xi^{*}-z\right\rangle+\left\langle n(\xi)-n\left(\xi^{*}\right), \xi^{*}-z\right\rangle\right) .
\end{aligned}
$$

In addition,

$$
\left|\left\langle n(\xi)-n\left(\xi^{*}\right), \xi^{*}-z\right\rangle\right| \lesssim\left|\xi-\xi^{*}\right|\left|\xi^{*}-z\right| \lesssim\left|\xi^{*}-z\right| \rho(\xi) .
$$

The domain $\Omega$ is convex; hence, $\operatorname{Re}\left\langle n\left(\xi^{*}\right), \xi^{*}-z\right\rangle \geq 0$ for $z \in \partial \Omega$ (see [10]). Assuming that the value $\left|\xi^{*}-z\right|$ is sufficiently small, we obtain the inequalities

$$
\begin{aligned}
|\langle\partial \rho(\xi), \xi-z\rangle| & \gtrsim\left(\left(\operatorname{dist}(\xi, \partial \Omega)+\operatorname{Re}\left\langle n\left(\xi^{*}\right), \xi^{*}-z\right\rangle\right)^{2}+\left(\operatorname{Im}\left\langle n\left(\xi^{*}\right), \xi^{*}-z\right\rangle\right)^{2}\right)^{1 / 2} \\
& \gtrsim \operatorname{dist}(\xi, \partial \Omega)+\left|\left\langle n\left(\xi^{*}\right), \xi^{*}-z\right\rangle\right| \gtrsim \rho(\xi)+v\left(\xi^{*}, z\right) ;
\end{aligned}
$$

the inverse inequality obviously follows from the triangle inequality. Due to the continuity of the considered expressions, local estimates can be transferred to arbitrary values $z \in \Omega$ and $\xi \in \mathbb{C}^{d} \backslash \Omega$.

The following lemma shows that the function $v(\xi, z)$ defines a quasimetric on $\partial \Omega$ (for details, see [7]). Note that the estimates hold locally, and this is compensated afterwards by a choice of finite pseudoanalytic continuations. 
Lemma 2.2. Let a domain $\Omega$ be strictly convex. Then the following estimates hold:

(1) $\operatorname{dist}(z, \partial \Omega) \asymp \rho(z) \asymp|z-\xi|^{2}, z \in T_{\xi} \bigcap \Omega_{2}, \xi \in \partial \Omega$;

(2) $|\xi-z|^{2} \lesssim v(\xi, z) \lesssim|\xi-z|, z, \xi \in \partial \Omega$;

(3) $v(\zeta, \xi) \asymp v(\xi, \zeta), \zeta, \xi \in \partial \Omega$;

(4) $v(\zeta, \xi) \lesssim v(\xi, w)+v(w, \zeta), \zeta, \xi, w \in \partial \Omega$;

(5) $|\xi-z|^{2} \lesssim v(\xi, z), \xi \in \Omega_{2} \backslash \Omega, z \in \partial \Omega$.

Proof. (1) Let $z \in T_{\xi} \cap \Omega_{2}$ and expand $\rho(z)$ by the Taylor formula:

$$
\begin{aligned}
\rho(z) & =\rho(\xi)+2 \operatorname{Re}\langle\partial \rho(\xi), z-\xi\rangle+\frac{1}{2} d^{2} \rho(\xi)\left[\frac{z-\xi}{|z-\xi|}\right]|z-\xi|^{2}+o\left(|z-\xi|^{2}\right) \\
& =\frac{1}{2} d^{2} \rho(\xi)\left[\frac{z-\xi}{|z-\xi|}\right] \cdot|z-\xi|^{2}+o\left(|z-\xi|^{2}\right) \asymp|z-\xi|^{2} .
\end{aligned}
$$

(2) Similarly to the previous item, by the Taylor formula,

$$
2 \operatorname{Re}\langle\partial \rho(\xi), z-\xi\rangle=-\frac{1}{2} d^{2} \rho(\xi)\left[\frac{z-\xi}{|z-\xi|}\right] \cdot|z-\xi|^{2}+o\left(|z-\xi|^{2}\right) \asymp|z-\xi|^{2},
$$

which gives us the lower bound. The upper bound is obvious.

(3) Estimate the value $|v(\xi, z) / v(z, \xi)|$ :

$$
\begin{aligned}
\left|\frac{\langle\partial \rho(\xi), \xi-z\rangle}{\langle\partial \rho(z), z-\xi\rangle}\right| & =\left|\frac{\langle\partial \rho(z), \xi-z\rangle-\langle\partial \rho(z)-\partial \rho(\xi), \xi-z\rangle}{\langle\partial \rho(z), z-\xi\rangle}\right| \\
& \lesssim 1+\frac{|\xi-z|^{2}}{|\xi-z|^{2}} \lesssim 1 .
\end{aligned}
$$

The inverse inequality is obtained by symmetry.

(4) This property is easily derived from the previous items:

$$
\begin{aligned}
|\langle\partial \rho(\zeta), \zeta-\xi\rangle| & \leq|\langle\partial \rho(\zeta), \zeta-w\rangle|+|\langle\partial \rho(\zeta)-\partial \rho(w), w-\xi\rangle|+|\langle\partial \rho(w), w-\xi\rangle| \\
& \lesssim v(\zeta, w)+v(w, \xi)+|\zeta-w||w-\xi| \\
& \lesssim v(\xi, w)+v(w, \zeta)+|\zeta-w|^{2}+|w-\xi|^{2} \lesssim v(\xi, w)+v(w, \zeta) .
\end{aligned}
$$

(5) By item (2) of this Lemma, $\left|\xi^{*}-z\right| \lesssim v\left(\xi^{*}, z\right)^{1 / 2}$, and

$$
|\xi-z| \leq\left|\xi-\xi^{*}\right|+\left|\xi^{*}-z\right| \lesssim \rho(\xi)+v\left(\xi^{*}, z\right)^{1 / 2} \lesssim \sqrt{\rho(\xi)+v\left(\xi^{*}, z\right)} \lesssim v(\xi, z)^{1 / 2}
$$

Note that the inverse inequality does not hold.

Lemma 2.3. There exists a constant $A=A(\rho)$ such that from the conditions $v(\xi, z)>A h$ and $v(z, w)<h$ it follows uniformly in $h>0$ that $v(\xi, z) \asymp v(\xi, w)$ for $\xi \in \Omega_{2} \backslash \Omega, z, w \in \partial \Omega$.

Proof. Note that since $v(\xi, z)$ defines a quasimetric on $\partial \Omega$, there exists a constant $A_{0}$ such that the statement of the lemma holds for $\xi \in \partial \Omega$. Now let $\xi \in \mathbb{C}^{d} \backslash \Omega$. Then by Lemma 2.2, there exists a constant $c>1$ such that

$$
c^{-1}\left(\rho(\xi)+v\left(\xi^{*}, z\right)\right) \leq v(\xi, z) \leq c\left(\rho(\xi)+v\left(\xi^{*}, z\right)\right) .
$$

Let $v(\xi, z)>2 c A_{0}$. Then either $\rho(\xi)>A_{0} h$ or $v\left(\xi^{*}, z\right)>A_{0} h$. Assume that the first inequality $\rho(\xi)>A_{0} h$ holds. Then

$$
\begin{aligned}
v(\xi, w) & \lesssim \rho(\xi)+v\left(\xi^{*}, w\right) \lesssim \rho(\xi)+v\left(\xi^{*}, z\right)+v(z, w) \\
& \lesssim \rho(\xi)+h+v\left(\xi^{*}, z\right) \lesssim \rho(\xi)+v\left(\xi^{*}, z\right) \lesssim v(\xi, z) .
\end{aligned}
$$

The inverse inequality is obtained in a similar way.

Now let $v\left(\xi^{*}, z\right)>A_{0} h$; then $v\left(\xi^{*}, z\right) \asymp v\left(\xi^{*}, w\right)$, and, hence,

$$
v(\xi, w) \asymp \rho(\xi)+v\left(\xi^{*}, w\right) \asymp \rho(\xi)+v\left(\xi^{*}, z\right) \asymp v(\xi, z) .
$$

The lemma is proved.

The main estimate of the kernel, which is used later, is stated as a corollary of Lemmas 2.2 and 2.3. 
Corollary 2.4. Let $z, w \in \partial \Omega$ and $\xi \in \Omega_{2} \backslash \Omega$, with $v(\xi, w)>A h$ and $v(z, w)<h$. Then the estimate

$$
|K(\xi, z)-K(\xi, w)| \lesssim \frac{v(z, w)^{1 / 2}}{v(\xi, z)^{d+1 / 2}}
$$

holds uniformly in $h>0$.

Proof. Note that $K(\xi, z)=\frac{1}{\langle\partial \rho(\xi), \xi-z\rangle^{d}}$. We have the relations

$$
\begin{aligned}
|\langle\partial \rho(\xi), \xi-z\rangle-\langle\partial \rho(\xi), \xi-w\rangle| & =|\langle\partial \rho(\xi), w-z\rangle| \leq|\langle\partial \rho(\xi)-\partial \rho(z), z-w\rangle| \\
& +|\langle\partial \rho(z), z-w\rangle| \lesssim|\xi-z||z-w|+v(z, w) \\
& \lesssim v(\xi, z)^{1 / 2} v(z, w)^{1 / 2}+v(z, w)^{1 / 2} v(\xi, w)^{1 / 2} \lesssim v(\xi, w)^{1 / 2} v(z, w)^{1 / 2}
\end{aligned}
$$

Therefore, taking into account that $v(\xi, z) \asymp v(\xi, w)$ by Lemma 2.3, we obtain the estimate

$$
|K(\xi, z)-K(\xi, w)| \lesssim \frac{v(\xi, w)^{1 / 2} v(z, w)^{1 / 2} v(\xi, w)^{d-1}}{v(\xi, z)^{d} v(\xi, w)^{d}} \lesssim \frac{v(z, w)^{1 / 2}}{v(\xi, z)^{d+1 / 2}}
$$

which proves the corollary.

2.3. Integral estimates of the kernel. Integral estimates of the kernel of the Cauchy-Leray-Fantappiè operator are based on the following lemma defining a system of special neighborhoods on $\partial \Omega$.

Lemma 2.5. Let $\xi \in \mathbb{C}^{d} \backslash \Omega$ and let $\delta>0$. Define

$$
V(\xi, \delta)=\{z \in \partial \Omega: v(\xi, z)<\delta\}
$$

then $\sigma V(\xi, \delta) \lesssim \delta^{d}$.

Proof. Let, as before, $\xi^{*}$ be the closest point of the boundary $\partial \Omega$ to the point $\xi$. Then, by Lemma $2.1, v(\xi, z) \lesssim$ $\rho(\xi)+v\left(\xi^{*}, z\right)$, and hence, it is sufficient to consider the case $\xi \in \partial \Omega$. But in this case, the result is well-known since the neighborhoods $V(\xi, \delta), \xi \in \partial \Omega$, are Hörmander ellipsoids.

Remark 2.6. Let $0 \leq r \leq 1, \xi \in \mathbb{C}^{d} \backslash \Omega, \delta>0$, and let

$$
V(\xi ; r, \delta)=\left\{z \in \partial \Omega_{r}: v(\xi, z)<\delta\right\}
$$

Then it follows from the smoothness of the function $\rho$ that $\sigma_{r} V(\xi ; r, \delta) \lesssim \delta^{d}$.

Lemma 2.7. Assume that $\alpha>0$ and $0<r<\delta<1$. Then

$$
I_{\alpha}(\xi, \delta)=\int_{\substack{z \in \partial \Omega, v(\xi, z)>\delta}} \frac{d \sigma(z)}{v(\xi, z)^{d+\alpha}} \lesssim \delta^{-\alpha}, \quad \xi \in \Omega_{2} \backslash \Omega,
$$

and

$$
J_{\alpha}(z, \delta)=\int_{\substack{\xi \in \partial \Omega_{r}, v(\xi, z)>\delta}} \frac{d \sigma_{r}(\xi)}{v(\xi, z)^{d+\alpha}} \lesssim \delta^{-\alpha}, \quad z \in \Omega
$$

Proof. We prove the first inequality. For this purpose, consider the sets

$$
V_{k}=\left\{z \in \partial \Omega: 2^{k} \delta \leq v(\xi, z) \leq 2^{k+1} \delta\right\}
$$

then $\{z \in \partial \Omega: v(\xi, z)>\delta\}=\bigcup_{k=1}^{\infty} V_{k}$ and $\sigma\left(V_{k}\right) \lesssim 2^{k d} \delta^{d}$. Hence,

$$
I_{\alpha}(z, \delta) \lesssim \sum_{k=1}^{\infty} \frac{2^{k d} \delta^{d}}{\left(2^{k} \delta\right)^{d+\alpha}} \lesssim \sum_{k=1}^{\infty} 2^{-k \alpha} \delta^{-\alpha} \lesssim \delta^{-\alpha}
$$

The second inequality is proved in a similar way by considering the sets $W_{k}=\left\{\xi \in \partial \Omega_{r}: 2^{k} \delta \leq v(\xi, z) \leq 2^{k+1} \delta\right\}$; we again take $\sigma_{r}\left(W_{k}\right) \lesssim 2^{k d} \delta^{d}$. 
Lemma 2.8. If $0<r<\delta<1$, then

$$
\int_{\substack{z \in \partial \Omega, v(\xi, z)<\delta}} \frac{d \sigma(z)}{v(\xi, z)^{d}} \lesssim 1+\log \frac{\delta}{r}, \quad \rho(\xi)=r,
$$

and

$$
\int_{\substack{\xi \in \partial \Omega_{r}, v(\xi, z)<\delta}} \frac{d \sigma_{r}(\xi)}{v(\xi, z)^{d}} \lesssim 1+\log \frac{\delta}{r}, \quad z \in \Omega .
$$

Proof. The proof of these inequalities is similar to the proof of the previous lemma; however, it is necessary to control the number of sets into which we partition the integration domain. Now let $V_{k}=\left\{z \in \partial \Omega: 2^{k} \leq\right.$ $\left.v(\xi, z) \leq 2^{k+1}\right\}, k \in \mathbb{Z}$, and let $\rho(\xi)=r<\delta$. Note that by Lemma 2.1,

$$
c^{-1} v(\xi, z) \leq \rho(\xi)+v\left(\xi^{*}, z\right) \leq c v(\xi, z)
$$

hence,

$$
c^{-1} 2^{k}-\delta \leq c^{-1} v(\xi, z)-\delta \leq v\left(\xi^{*}, z\right) \leq c v(\xi, z)-r \leq c 2^{k+1}-r, \quad z \in V_{k} .
$$

Therefore, since $0 \leq v\left(\xi^{*}, z\right) \leq \delta$, we conclude that $V_{k}=\varnothing$ if $2^{k+1}<c^{-1} r$ or $2^{k}>c \cdot \delta$, i.e., for $k<k_{1}(r)=$ $\log _{2} c^{-1} r-1$ and for $k>k_{2}(\delta)=\log _{2} c \delta$. Thus,

$$
\{z \in \partial \Omega: v(\xi, z)<\delta\}=\bigcup_{k_{1} \leq k \leq k_{2}} V_{k}
$$

and

$$
\begin{aligned}
\int_{\substack{\xi \in \partial \Omega_{r}, v(\xi, z)<\delta}} \frac{d \sigma_{r}(\xi)}{v(\xi, z)^{d}} & \leq \sum_{k_{1} \leq k \leq k_{2}} \int_{V_{k}} \frac{d \sigma_{r}(\xi)}{v(\xi, z)^{d}} \\
& \lesssim \sum_{k_{1} \leq k \leq k_{2}} \frac{2^{k d}}{2^{(k+1) d}} \lesssim k_{2}(\delta)-k_{1}(\delta) \lesssim 1+\log \frac{\delta}{r} .
\end{aligned}
$$

The second inequality is proved in a similar way.

2.4. Approximation of the Cauchy-Leray-Fantappiè kernel. Corollary 2.4 provides an opportunity to local approximations of the Cauchy-Leray-Fantappiè kernel (see Sec. 5.1). It is often required to construct a global approximation, which is possible due to Lemma 2.10 based on the V. K. Dzyadyk theorem on estimation of the Cauchy kernel (Theorem 1 in Sec. 1 of Chap. 7 of the monograph [4]). The approximation here is chosen similarly to that of [9].

Lemma 2.9. Assume that a domain $\Omega$ is strictly convex and $0 \in \Omega$. Then for an arbitrary point $\xi \in \partial \Omega$, the values of the expression $\lambda=\frac{\langle\partial \rho(\xi), z\rangle}{\langle\partial \rho(\xi), \xi\rangle}$ for $z \in \Omega$ belong to the domain $L(t)$ bounded by the bigger arc of the circle $|\lambda|=R=R(\Omega)$ and the chord $\left\{\lambda \in \mathbb{C}: \lambda=1+e^{i t} s, s \in \mathbb{R},|\lambda| \leq R\right\}$, where $t=\frac{\pi}{2}-\arg (\langle\partial \rho(\xi), \xi\rangle)$.

Proof. Let $\xi \in \partial \Omega$ and define

$$
\Lambda(\xi)=\left\{\lambda \in \mathbb{C}: \lambda=\frac{\langle\partial \rho(\xi), z\rangle}{\langle\partial \rho(\xi), \xi\rangle}, z \in \Omega\right\} .
$$

Note that since the domain $\Omega$ is convex and $0 \in \Omega$, the inequalities

$$
|\langle\partial \rho(\xi), \xi\rangle| \gtrsim|\partial \rho(\xi)||\xi| \gtrsim 1
$$

and

$$
\operatorname{Re}\langle\partial \rho(\xi), z-\xi\rangle \leq 0, \quad z \in \bar{\Omega}, \xi \in \partial \Omega .
$$

hold. The domain $\Lambda(\xi)$ is convex and contains 0 ; hence, the equality

$$
\frac{\langle\partial \rho(\xi), z\rangle}{\langle\partial \rho(\xi), \xi\rangle}=1+\frac{\langle\partial \rho(\xi), z-\xi\rangle}{\langle\partial \rho(\xi), \xi\rangle}
$$

with estimates (2.8) and (2.9) completes the proof of the lemma. 
Lemma 2.10. Assume that $\alpha>0$. Then for any $n=1,2, \ldots$ there exists a polynomial $K_{n}^{\text {glob }}(\xi, z)$ in $z$ of degree not exceeding $n$ such that for $z \in \partial \Omega$ and $\xi \in \mathbb{C}^{d} \backslash \Omega$, the estimates

$$
\left|K(\xi, z)-K_{n}^{\text {glob }}(\xi, z)\right| \lesssim \frac{1}{n^{\alpha}} \frac{1}{v(\xi, z)^{d+\alpha}}, \quad v(\xi, z) \geq \frac{1}{n} ;
$$

and

hold.

$$
\left|K_{n}^{\text {glob }}(\xi, z)\right| \lesssim n^{d}, \quad v(\xi, z) \leq \frac{1}{n}
$$

Proof. Note that due to [4] and [9], for any $n \in \mathbb{N}$ there exists a polynomial $T_{n}(t, \lambda)$ in $\lambda$ of degreee $n$ such that

$$
\left|\frac{1}{1-\lambda}-T_{n}(t, \lambda)\right| \lesssim \frac{1}{n^{\alpha}} \frac{1}{|1-\lambda|^{1+\alpha}}
$$

for $\lambda \in L(t) \backslash\left\{\lambda:|1-\lambda|<\frac{1}{n}\right\}$ such that coefficients of the polynomial $T_{n}(t, \lambda)$ continuously depend on the parameter $t$. Note also that by the maximum principle,

$$
T_{n}(t, \lambda) \lesssim n^{-1}, \quad \lambda \in L(t) \bigcap\left\{\lambda:|1-\lambda|<\frac{1}{n}\right\} .
$$

Setting

$$
K_{n d}^{\text {glob }}(\xi, z)=\frac{1}{\langle\partial \rho(\xi), \xi\rangle^{d}} T_{n}^{d}\left(t(\xi), \frac{\langle\partial \rho(\xi), z\rangle}{\langle\partial \rho(\xi), \xi\rangle}\right),
$$

it is easily seen that the polynomials $K_{n d}^{\text {glob }}(\xi, \cdot)$ satisfy relations $(2.10)-(2.11)$. Adding to the sequence of the polynomials the relation $K_{n}^{\text {glob }}=K_{d[n / d]}^{\text {glob }}$, we obtain the required approximation.

\section{Local approximations And the spaces $B_{p q}^{s}(\partial \Omega)$}

The definition of the Besov space given in Sec. 1 appears to be inconvenient in application to approximation problems. For this reason, the definition of modulus of continuity in terms of local polynomial approximations is often used. Note that the results of this section hold in the general case for an arbitrary domain with $C^{[s]+1}$ smooth boundary.

Let $K \subseteq \partial \Omega$ and $f \in L^{1}(K)$. Define the best approximation of the function $f$ by polynomials of degree $m$ in the metric of $L^{p}$ by the formula

$$
E_{m}(f, K)_{p}=\inf _{T \in \mathbb{P}_{m}}\left(\int_{K}|f-T|^{p} d \sigma\right)^{1 / p},
$$

where $\mathbb{P}_{m}$ is the set of all polynomials of degree $m$ in each variable, $m \geq 0$.

Remark 3.1. Note that the lower bound in definition (3.1) can be searched among polynomials $T$ such that $\|T\|_{L^{p}(K)} \leq 2\|f\|_{L^{p}(K)}$. In addition, by the equivalence of norms in a finite-dimensional space, the relation $\|T\|_{C^{\infty}(K)} \leq \frac{c(m)}{\sigma(K)}\|T\|_{L^{p}(K)}, T \in \mathbb{P}_{m}$, holds.

Consider the following special atlas of the boundary of the domain $\Omega$ :

$$
\partial \Omega=\bigcup_{k=1}^{N} \widetilde{Q}_{k}, \text { where } \widetilde{Q}_{k}=\varphi_{k}(Q), \text { and } Q=[0,1]^{2 d-1} \text { is the unit cube in } \mathbb{R}^{2 d-1}
$$

and assume that a partition of unity $\sum_{k=1}^{N} \chi_{k}=1$ is chosen such that

for some $0<\varepsilon<1$.

$$
\widetilde{Q}_{k, \varepsilon}=\varphi_{k}((1-\varepsilon) Q) \subset \operatorname{supp} \chi_{k} \subset \widetilde{Q}_{k}
$$

Denote by $Q_{h}=Q_{h}(x)=\left\{x+[0, h]^{2 d-1}\right\}$ the cube in $\mathbb{R}^{2 d-1}$ with side length $h$ and denote by $\widetilde{Q}_{h}=\varphi_{k}\left(Q_{h}\right)$ its image under the action of a diffeomorphism $\varphi_{k}$. The notation $\widetilde{Q}_{h / 2} \subset F \subset \widetilde{Q}_{h}$ formally means that there exist two points $x, y \in \mathbb{R}^{2 d-1}$ such that $\varphi_{k}\left(Q_{h / 2}(x)\right) \subset F \subset \varphi_{k}\left(Q_{h}(y)\right)$ for one of the functions $\varphi_{k}$. A similar notation is used when we use projections on tangent hyperplanes instead of diffeomorphisms (see Remark 3.5). 
Definition 3.2. Let $f \in L^{p}(\partial \Omega)$; define a polynomial modulus of smoothness of order $m \geq 1$ by the formula

$$
\omega_{m}(f, h)_{p}=\sup \left(\sum_{j=1}^{N} E_{m-1}\left(f, F_{j}\right)_{p}^{p}\right)^{1 / p},
$$

where the upper bound is taken over all partitions $\left\{F_{j}\right\}$ of the boundary of the domain $\Omega$ such that $\widetilde{Q}_{h / 2} \subset F_{j} \subset$ $\widetilde{Q}_{h}$.

Note that for cubes, the equivalence of the definition of modulus of continuity in terms of approximations and differences (see Definitions (1.2)-(1.6)) is proved in [3], and we rely on the following lemma.

Lemma 3.3 (Brudnyı̆ and Irodova [3]). Assume that $m \geq 1$ and $0<p \leq \infty$. Then the equivalence

$$
\omega^{m}(f, t)_{p} \asymp \sup \left(\sum_{j=1}^{N} E_{m-1}\left(f, Q_{t}^{j}\right)_{p}^{p}\right)^{1 / p}
$$

holds uniformly in $f \in L^{p}\left([0,1]^{l}\right)$ and $t \in[0,1]$ where the upper bound is taken over all the sets $\left\{Q_{t}^{j}\right\}$ of disjoint cubes with side length $t$.

Theorem 3.4. Assume that $1 \leq p, q \leq \infty$ and $s>0$, let a domain $\Omega$ have a smooth boundary, and let $f \in L^{p}(\partial \Omega)$. Then $f \in B_{p q}^{s}(\partial \Omega)$ if and only if

$$
\widetilde{c}_{p q}(f)=\left(\int_{0}^{1}\left(\frac{\omega_{m}(f, t)_{p}}{t^{s}}\right)^{q} \frac{d t}{t}\right)^{1 / q}<\infty
$$

for $q<\infty$ and

$$
\widetilde{c}_{p q}(f)=\sup _{t>0} \frac{\omega_{m}(f, t)_{p}}{t^{s}}<\infty
$$

for $q=\infty$, where $m \in \mathbb{N}$ and $m>s$. In addition, $\|f\|_{B_{p q}^{s}} \asymp\|f\|_{p}+\widetilde{c}_{p q}(f)$.

Proof. Consider the atlas and partition of unity defined in relations (3.2) and (3.3). The idea of the proof is to assign to polynomials which approximate the function $f$ polynomials wich approximate the functions $\left(f \chi_{k}\right) \circ \varphi_{k}$, and vice versa. There is the equivalence of the definitions on the cube $Q$, and, by Lemma 3.3, for $m \geq 0$ we have the equivalence

$$
\omega^{m+1}\left(\left(f \chi_{k}\right) \circ \varphi_{k}, t\right)_{p} \asymp \sup \left(\sum_{j} E_{m}\left(\left(f \chi_{k}\right) \circ \varphi_{k}, Q_{t}^{j}\right)_{p}^{p}\right)^{1 / p} .
$$

By means of this equivalence, we prove the following two estimates, which imply the statement of the theorem:

$$
\omega^{m+1}\left(\left(f \chi_{k}\right) \circ \varphi_{k}, t\right)_{p} \lesssim \omega_{m+1}(f, t)_{p}+\|f\|_{L^{p}(\partial \Omega)} t^{m+2}
$$

and

$$
\omega_{m+1}(f, t)_{p} \lesssim \sum_{k=1}^{N} \omega^{m+1}\left(\left(f \chi_{k}\right) \circ \varphi_{k}, t\right)_{p}+\|f\|_{L^{p}(\partial \Omega)} t^{m+2} .
$$

We prove inequality (3.5). For this purpose, fix a value $k$, consider a family of disjoint cubes $\left\{Q_{t}^{j}\right\}$ with side length $t$ that are contained in the unit cube $Q_{1}$, and assign to each cube its image in $\partial \Omega$ under the action of the diffeomorphism $\varphi_{k}$. Let $\widetilde{Q}_{t}^{j}=\varphi_{k}\left(Q_{t}^{j}\right)$.

Consider a polynomial $\widetilde{P_{m}}$ of coordinate degree $m$ defined on $\widetilde{Q}_{t}^{j}$ such that $\left\|\widetilde{P}_{m}\right\|_{L^{p}\left(\widetilde{Q}_{t}^{j}\right)} \leq 2\|f\|_{L^{p}\left(\widetilde{Q}_{t}^{j}\right)}($ see Remark 3.1). Then the function $\left(\widetilde{P}_{m} \chi_{k}\right) \circ \varphi_{k}$ is smooth on $Q_{t}^{j}$, and by the Taylor formula, there exists a polynomial $P_{m}$ of degree $m$ defined on $Q_{t}^{j}$ such that for $y \in Q_{t}^{j}$, the estimates

hold.

$$
\left|\left(\widetilde{P}_{m} \chi_{k}\right) \circ \varphi_{k}(y)-P_{m}(y)\right| \lesssim\left\|\widetilde{P}_{m} \chi_{k} \circ \varphi_{k}\right\|_{C^{\infty}\left(Q_{t}^{j}\right)} t^{m+1} \lesssim \frac{\|f\|_{L^{p}\left(\widetilde{Q}_{t}^{j}\right)}}{\left|\widetilde{Q}_{t}^{j}\right|} t^{m+1}
$$


Therefore,

$$
\begin{aligned}
\left\|\left(f \chi_{k}\right) \circ \varphi_{k}-P_{m}\right\|_{L^{p}\left(Q_{t}^{j}\right)} & \lesssim\left\|\left(f-\widetilde{P}_{m}\right) \chi_{k} \circ \varphi_{k}\right\|_{L^{p}\left(Q_{t}^{j}\right)}+\|f\|_{L^{p}\left(\widetilde{Q}_{t}^{j}\right)} t^{m+1} \\
& \leq\left\|f-\widetilde{P}_{m}\right\|_{L^{p}\left(\widetilde{Q}_{t}^{j}\right)}+\|f\|_{L^{p}\left(\widetilde{Q}_{t}^{j}\right)} t^{m+1} .
\end{aligned}
$$

Due to the arbitrariness of the choice of the polynomial $\widetilde{P}_{m}$, we have the inequalities

$$
E_{m}\left(\left(f \chi_{k}\right) \circ \varphi_{k}, Q_{t}^{j}\right)_{p} \lesssim E_{m}\left(f, \widetilde{Q}_{t}^{j}\right)_{p}+\|f\|_{L^{p}\left(\widetilde{Q}_{t}^{j}\right)} t^{m+1}
$$

and

$$
\left(\sum_{j} E_{m}\left(\left(f \chi_{k}\right) \circ \varphi_{k}, Q_{t}^{j}\right)_{p}^{p}\right)^{1 / p} \lesssim\left(\sum_{j} E_{m}\left(f, \widetilde{Q}_{t}^{j}\right)_{p}^{p}\right)^{1 / p}+\|f\|_{L^{p}\left(\widetilde{Q}_{k}\right)} t^{m+1} \leq \omega_{m+1}(f, t)_{p}+\|f\|_{L^{p}\left(\widetilde{Q}_{k}\right)} t^{m+1} .
$$

Passing to the upper bound in the left-hand side of this inequality, we obtain the inequality

$$
\omega^{m+1}\left(\left(f \chi_{k}\right) \circ \varphi_{k}, t\right)_{p} \lesssim \omega_{m+1}(f, t)_{p}+\|f\|_{L^{p}\left(\widetilde{Q}_{k}\right)} t^{m+1} .
$$

To prove inequality (3.6), we use the fact that the supports of functions from the partition of unity cover $\partial \Omega$, and we may assume that $\partial \Omega=\bigcup_{k=1}^{N} \widetilde{Q}_{k, \varepsilon}$. Assume that the value $t>0$ is sufficiently small to choose a covering of the cube $(1-\varepsilon) Q_{1}$ by disjoint cubes with side length $t$, and let

$$
(1-\varepsilon) Q \subset \bigcup Q_{t}^{j} \subset Q
$$

Consider a polynomial $P_{m}$ of coordinate degree $m$ defined on $Q_{t}^{j}$. Note that $\left\|1 / \chi_{k}\right\|_{C^{\infty}\left(\widetilde{Q}_{k, \varepsilon}\right)}<\infty$ and the function $\left(P_{m} / \chi_{k}\right) \circ \psi_{k}$ is smooth on $\widetilde{Q}_{k, \varepsilon}$. If parameter $t>0$ is sufficiently small, then, similarly to the previous case, there exists a polynomial $\widetilde{P}_{m}$ of degree $m$ such that

$$
\left\|\left(P_{m} / \chi_{k}\right) \circ \psi_{k}-\widetilde{P}_{m}\right\|_{L^{p}\left(\widetilde{Q}_{t}^{j}\right)} \lesssim\|f\|_{L^{p}\left(\widetilde{Q}_{t}^{j}\right)} t^{m+1}
$$

hence,

$$
\begin{aligned}
\left\|f-\widetilde{P}_{m}\right\|_{L^{p}\left(\widetilde{Q}_{t}^{j}\right)} & \lesssim\left\|f-\left(P_{m} / \chi_{k}\right) \circ \psi_{k}\right\|_{L^{p}\left(\widetilde{Q}_{t}^{j}\right)}+\|f\|_{L^{p}\left(\widetilde{Q}_{t}^{j}\right)} t^{m+1} \\
& \lesssim\left\|\left(f \chi_{k}\right) \circ \varphi_{k}-P_{m}\right\|_{L^{p}\left(\widetilde{Q}_{t}^{j}\right)}+\|f\|_{L^{p}\left(\widetilde{Q}_{t}^{j}\right)} t^{m+1}
\end{aligned}
$$

Therefore,

$$
\begin{aligned}
\left(\sum_{j} E_{m}\left(f, \widetilde{Q}_{t}^{j}\right)_{p}^{p}\right)^{1 / p} & \lesssim\left(\sum_{j} E_{m}\left(\left(f \chi_{k}\right) \circ \varphi_{k}, Q_{t}^{j}\right)_{p}^{p}\right)^{1 / p}+\|f\|_{L^{p}\left(\widetilde{Q}_{k}\right)} t^{m+1} \\
& \lesssim \omega^{m+1}\left(\left(f \chi_{k}\right) \circ \varphi_{k}, t\right)_{p}+\|f\|_{L^{p}\left(\widetilde{Q}_{k}\right)} t^{m+1} .
\end{aligned}
$$

It remains to improve the left-hand side of this inequality before we obtain the required estimate. Let $\partial \Omega=\bigcup_{j=1}^{N} F_{j}$ with $\widetilde{Q}_{j, t / 2} \subset F_{j} \subset \widetilde{Q}_{j, t}$. Note that the cube $\widetilde{Q}_{j, t}$ assigned to the set $F_{j}$ can intersect only a finite number of cubes assigned to the remaining sets $F_{l}$; in particular, $\#\left\{k: \widetilde{Q}_{j, t} \cap \widetilde{Q}_{k, t} \neq \varnothing\right\} \leq 4^{d}$. Hence,

$$
\begin{aligned}
\omega_{m+1}(f, t)_{p} & =\sup \left(\sum_{j} E_{m}\left(f, F_{j}\right)_{p}^{p}\right)^{1 / p} \lesssim \sup \left(\sum_{j} E_{m}\left(f, \widetilde{Q}_{j, t}\right)_{p}^{p}\right)^{1 / p} \\
& \lesssim \sum_{k=1}^{N} \omega^{m+1}\left(\left(f \chi_{k}\right) \circ \varphi_{k}, t\right)_{p}+\|f\|_{L^{p}(\partial \Omega)} t^{m+1} .
\end{aligned}
$$

Therefore, inequalities (3.5) and (3.6) are proved, and the proof of the theorem is completed. 
Remark 3.5. Note that in the definition of the value $\omega_{m}(f, h)_{p}$ we can use partitions into sets $F_{j}$ such that $Q_{h / 2}\left(\zeta_{1}\right) \subset \operatorname{pr}_{\xi} F_{j} \subset Q_{h}\left(\zeta_{2}\right)$ for some point $\xi \in \partial \Omega$ and points $\zeta_{1}, \zeta_{2} \in T_{\xi}^{\mathbb{R}}$, where $\operatorname{pr}_{\xi}$ is the projection on the tangent plane at a point $\xi \in F_{j}$. This is possible due to the smoothness of the boundary $\partial \Omega$ and since $\left\|\operatorname{pr}_{\xi_{1}} \circ \operatorname{pr}_{\xi_{2}}^{-1}\right\|_{C^{\infty}}<c(\Omega)<\infty$ for any points $\xi_{1}, \xi_{2} \in \partial \Omega$. In the following, we use this definition of the value $\widetilde{c}_{p q}(f)$.

\section{Construction of almost best local approximation}

The result of the previous section allows us to pass from the concepts of the operator of taking differences best local approximations. In this section, we consider an interpolating construction whicht provides an almost best approximation on a set $K \subset \partial \Omega$ such that $Q_{h} \subset \operatorname{pr}_{\xi}(K) \subset Q_{2 h}$ for some point $\xi \in \partial \Omega$ and $h>0$. Let $P_{0}$ be a polynomial of degree $m$ of one real variable such that

$$
\int_{0}^{1} P_{0}(x) d x=1 \quad \text { and } \quad \int_{0}^{1} x^{k} P_{0}(x) d x=0, \quad k=1, \ldots, m,
$$

and let $P_{1}(z)$ be a polynomial of degree $m$ of one complex variable such that

$$
\int_{Q} P_{1}(z) d \mu_{2}(z)=1 \text { and } \int_{Q} z^{k} P_{1}(z) d \mu_{2}(z)=0, \quad k=1, \ldots, m,
$$

where $Q=\{z \in \mathbb{C}: z=x+i y, x, y \in[0,1]\}$ is the unit cube in $\mathbb{C}$ and $\mu_{2}$ is the Lebesgue measure in $\mathbb{C}$.

Let $z \in \mathbb{C}^{d}$; introduce the following notation: $z=\left(z^{\prime}, w\right)$, where $z^{\prime}=\left(z_{1}, \ldots, z_{d-1}\right) \in \mathbb{C}^{d-1}, w \in \mathbb{C}$.

Fix a point $\xi \in \partial \Omega$. By an analytically-linear change of coordinates we can take $\xi=0$ and

$$
T_{\xi}^{\mathbb{R}}=\left\{z \in \mathbb{C}^{d}: \operatorname{Im} z_{d}=0\right\}=\left\{z \in \mathbb{C}^{d}: z_{d} \in \mathbb{R}\right\}
$$

Then in this notation,

$$
\operatorname{pr}_{\xi}(z)=\left(z^{\prime}, \operatorname{Re} w\right) \text { and } \pi_{\xi}(z)=\left(z^{\prime}, 0\right),
$$

where, as before, $\mathrm{pr}_{\xi}$ and $\pi_{\xi}$ are the projectors on the tangent and complex tangent hyperplanes, respectively.

Let $u \in T_{\xi}^{\mathbb{R}}$ and $h, h_{1}>0$. Consider a parallelepiped in the tangent hyperplane:

$$
\widetilde{J}_{u}=u+\left\{z \in \mathbb{C}^{d}: z=\left(z^{\prime}, w\right), z^{\prime} \in[0, h]^{2 d-2} \subset \mathbb{C}^{d-1}, w=z_{d-1}, \operatorname{Re} w \in\left[0, h_{1}\right], \operatorname{Im} w=0\right\} .
$$

Since the domain is regular, the projection operator on the tangent hyperplane is locally invertible in a neighborhood of the point $\xi$. Assuming that the parallelepiped $\widetilde{J}_{u}$ is sufficiently small and close to the point $\xi$, denote

$$
J_{u}=\operatorname{pr}_{\xi}^{-1}\left(\widetilde{J}_{u}\right), \quad\left(u^{\prime}, w_{0}\right)=\operatorname{pr}_{\xi}^{-1}(u), \quad\left(z^{\prime}, w_{1}\left(z^{\prime}\right)\right)=\operatorname{pr}_{\xi}^{-1}\left(z^{\prime}, u_{d}+h_{1}\right),
$$

where $\left(z^{\prime}, 0\right) \in \pi_{\xi}(J)$, and, obviously, $\operatorname{Re} w_{0}=u_{d}$ and $\operatorname{Re} w_{1}\left(z^{\prime}\right)=u_{d}+h_{1}$.

In addition, consider the line segment connecting the points $u$ and $\left(z^{\prime}, u_{d}+h_{1}\right)$ and the curve $\widetilde{\gamma}_{z^{\prime}}$ connecting the points $\left(u^{\prime}, w_{0}\right)$ and $\left(z^{\prime}, w_{1}\left(z^{\prime}\right)\right)$, which is obtained as the image of this line segment under the inverse projection $\operatorname{pr}_{\xi}^{-1}$ on the boundary of the domain $\Omega$, i.e.,

$$
\widetilde{\gamma}_{z^{\prime}}=\operatorname{pr}_{\xi}^{-1}\left\{(1-t) u+t\left(z^{\prime}, u_{d}+h_{1}\right)\right\}
$$

Finally, assign to the curve $\widetilde{\gamma}_{z^{\prime}}$ the curve $\gamma_{z^{\prime}}$ in the complex plane which is defined by the last coordinate of the curve $\widetilde{\gamma}_{z^{\prime}}$. Introduce the notation $J_{u}^{\prime}=\pi_{\xi}\left(J_{u}\right)$.

Define the function

$$
P_{J_{u}}(z)=P_{J_{u}}\left(z^{\prime}, w\right)=\frac{1}{h^{2(d-1)}} \frac{1}{w_{1}\left(z^{\prime}\right)-w_{0}} P_{d}\left(\frac{z^{\prime}-u^{\prime}}{h}\right) P_{0}\left(\frac{w-w_{0}}{w_{1}\left(z^{\prime}\right)-w_{0}}\right),
$$

where $P_{d}\left(z^{\prime}\right)=P_{1}\left(z_{1}\right) \cdot \ldots \cdot P_{1}\left(z_{d-1}\right)$.

This is not a polynomial and even not an analytic function but this function reconstructs the value of any polynomial whose coordinate degree does not exceed $m$ at a point $z_{0}=\left(u^{\prime}, w_{0}\right) \in \partial \Omega$ by the following operation: 


$$
\begin{aligned}
\int_{J_{u}^{\prime}} d \mu_{2 d-2}\left(z^{\prime}\right) \int_{\gamma_{z^{\prime}}} & T\left(z^{\prime}, w\right) P_{J_{u}}\left(z^{\prime}, w\right) d w \\
= & \int_{J_{u}^{\prime}} \frac{d \mu_{2 d-2}\left(z^{\prime}\right)}{h^{2 d-2}} P_{d}\left(\frac{z^{\prime}-u^{\prime}}{h}\right) \int_{\gamma_{z^{\prime}}} T\left(z^{\prime}, w\right) P_{0}\left(\frac{w-w_{0}}{w_{1}\left(z^{\prime}\right)-w_{0}}\right) \frac{d w}{w_{1}\left(z^{\prime}\right)-w_{0}} \\
= & \int_{J_{u}^{\prime}} \frac{d \mu_{2 d-2}\left(z^{\prime}\right)}{h^{2(d-1)}} P_{d}\left(\frac{z^{\prime}-u^{\prime}}{h}\right) \int_{0}^{1} T\left(z^{\prime}+z_{0}^{\prime}, w_{0}+v\left(w_{1}\left(z^{\prime}\right)-w_{0}\right)\right) P_{0}(v) d v \\
& =\int_{J_{u}^{\prime}} P_{d}\left(\frac{z^{\prime}-u^{\prime}}{h}\right) T\left(z^{\prime}, w_{0}\right) \frac{d \mu_{2 d-2}\left(z^{\prime}\right)}{h^{2(d-1)}}=T\left(u^{\prime}, w_{0}\right)=T\left(z_{0}\right) .
\end{aligned}
$$

Passing from the curve $\gamma_{z^{\prime}}$ connecting the points $w_{0}$ and $w_{1}\left(z^{\prime}\right)$ to the integral over the segment line $[0,1]$ is possible by the change of variables $v=\frac{w-w_{0}}{w_{1}\left(z^{\prime}\right)-w_{0}}$ and the fact that the integrand is analytic. Note that the integration is performed over the half of the parallelepiped projection $\widetilde{J}_{u}$, which is swept by the curves $\widetilde{\gamma}_{z^{\prime}}$, with $|d w| d \mu_{2 d-2}\left(z^{\prime}\right) \asymp d \sigma\left(z^{\prime}, w\right)$.

We finally describe the operator of local almost best approximation. Let $K$ be a set such that $Q_{h}\left(\zeta_{1}\right) \subset$ $\operatorname{pr}_{\xi}(K) \subset Q_{2 h}\left(\zeta_{2}\right)$ for some points $\zeta_{1}, \zeta_{2} \in T_{\xi}$ and some function $f \in L^{1}(K)$. Consider the partition of the cube $Q_{h}=Q_{h}^{d-1} \times[0, h]$ into shifts of parallelepipeds of the form

$$
Q^{(m)}=\left\{\left(z^{\prime}, w\right): x_{j}, y_{j} \in\left[0, h /(\sqrt{m+1}+1)^{2}+1\right], x_{d} \in[0, h / m]\right\} \subset T_{\xi}
$$

and decompose the cube $Q_{h}(\zeta)$ in a similar way. Enumerate elements of partition arbitrarily, e.g., diagonally, then $\bigcup_{j=1}^{(m+1)^{d}} \widetilde{Q}_{j}\left(z_{j}\right) \subset K$, where $\widetilde{Q}_{j}\left(z_{j}\right)$ are the inverse projections of the corresponding elements of the partition. Let $Q_{j}^{\prime}=\pi_{\xi}\left(\widetilde{Q}_{j}\left(z_{j}\right)\right)$; denote by $\mathcal{P}_{K}(f)$ a polynomial such that

$$
\mathcal{P}_{K}(f)\left(z_{j}\right)=\int_{Q_{j}^{\prime}} d \mu_{2 d-2}\left(z^{\prime}\right) \int_{\gamma_{z^{\prime}}} P_{Q_{j}\left(z_{j}\right)}\left(z^{\prime}, w\right) f\left(z^{\prime}, w\right) d w, j=1, \ldots,(m+1)^{d} .
$$

Then $\mathcal{P}_{K}$ is the projection on the space of polynomials whose coordinate degrees do not exceed $m$, and $\mathcal{P}_{K}(T)=T$ for any polynomial whose degree by every coordinate does not exceed $m$. In addition

$$
\max _{\operatorname{dist}(z, K) \leq \lambda h}\left|\mathcal{P}_{K}(f)(z)\right| \leq \frac{c}{\sigma(K)} \int_{K}|f| d \sigma,
$$

since $\max _{z \in Q_{\lambda h}}\left|P_{Q_{h}}(z)\right|<\frac{c(\lambda, \Omega)}{h^{2 d-1}}$; the constant $c$ depends only on parameter $\lambda>0$ and $m \in \mathbb{N} \cup\{0\}$, and the domain $\Omega$, and does not depend on $K$ and $f$. This implies the estimate

$$
\left\|f-\mathcal{P}_{K} f\right\|_{L^{p}(K)} \leq(c+1) E_{m}(f, K)_{p} ;
$$

hence, $\mathcal{P}_{K} f$ can be used instead of the best approximation polynomial.

Remark 4.1. Assume that $f \in H^{p}(\partial \Omega)$ and that $\partial \Omega=\bigcup_{j=1}^{N} F_{j}$, where the sets $F_{j}$ are such that $Q_{h} \subset \operatorname{pr}_{\xi}\left(F_{j}\right) \subset$ $Q_{2 h}$. Define a piecewise-polynomial function $T_{h}$ that coincides on $F_{j}$ with the polynomial $\mathcal{P}_{F_{j}}$; then

$$
\omega_{m}(f, h)_{p} \asymp \sup \left\|f-T_{h}\right\|_{L^{p}(\partial \Omega)},
$$

where the upper bound is taken over all the partitions of this form.

Remark 4.2. Note a useful inequality which connects local polynomial approximations for different parameters $p$ :

$$
E_{m}(f, K)_{1} \leq \sigma(K)^{1-\frac{1}{p}} E_{m}(f, K)_{p} .
$$




\section{Two METhODS OF PSEUdOANALYTIC CONTINUATION}

5.1. Continuation by local approximations. Let $f \in H^{1}(\Omega)$, let $m$ be an integer, and let $z \in \mathbb{C}^{d} \backslash \Omega$. Set

$$
E(f, z)=E_{m}(f, J(z))_{1},
$$

where

$$
J(z)=\left\{\xi \in \partial \Omega: \pi_{z *}(\xi) \in Q_{\rho(z) / 10}\left(z^{*}\right)\right\}
$$

Theorem 5.1. Assume that

$$
E(f, z) \rho(z)^{-2 d} \in L^{p}\left(\mathbb{C}^{d} \backslash \Omega\right)
$$

for some $p \geq 1$. Then $f \in L^{p}(\partial \Omega)$ and there exists a pseudoanalytic continuation $\mathbf{f}$ of the function $f$ such that

$$
|\bar{\partial} \mathbf{f}(z)| \lesssim E(f, z) \rho(z)^{-2 d}
$$

Proof. To begin with, we show that $f \in L^{p}(\partial \Omega)$, assuming that $p>1$. Let $J \subset \partial \Omega$ be a set such that $Q_{h} \subset \operatorname{pr}_{\xi} J \subset Q_{2 h}$ for some point $\xi \in \partial \Omega$ and some value $h>0$. We assign to this set a domain in $\mathbb{C}^{d} \backslash \Omega$ :

$$
L(J)=\left\{z \in \mathbb{C}^{d} \backslash \Omega: \operatorname{dist}(z, J)<h / 10, \operatorname{dist}(z, \partial \Omega)>h / 100\right\} .
$$

It is obvious that $\mu_{2 d}(L(J)) \asymp h^{2 d}$ and $E(f, z) \geq E_{m}(f, J)_{1}, z \in L(J)$.

Consider a sequence of the partitions of the boundary of the domain $\Omega$ :

$$
\partial \Omega=\bigcup_{k=1}^{2^{d n}} J_{k}^{n}, \quad \operatorname{diam}\left(J_{k}^{n}\right) \asymp 2^{-n}, \quad \sigma J_{k}^{n} \asymp 2^{-(2 d-1) n},
$$

where each successive partition is obtained from the previous one by dividing its elements into $2^{d}$ parts.

Define a function $T_{n}$ on $\partial \Omega$ by setting $T_{n}(z)=\mathcal{P}_{J_{k}^{n}} f(z)$ for $z \in J_{k}^{n}$, where $\mathcal{P}_{J_{k}^{n}}$ is the polynomial projection defined in the previous section. Then $f=T_{n_{0}}+\sum_{n=n_{0}}^{\infty}\left(T_{n+1}-T_{n}\right)$ almost everywhere on $\partial \Omega$, and it remains to verify that $\sum_{n=n_{0}}^{\infty}\left\|T_{n+1}-T_{n}\right\|_{L^{p}(\partial \Omega)}<\infty$.

Note that for $z \in J_{k}^{n+1}$, the relations

$$
\begin{aligned}
\left|T_{n+1}(z)-T_{n}(z)\right| & =\left|\mathcal{P}_{J_{k}^{n+1}} f(z)-\mathcal{P}_{J_{k}^{n}} f(z)\right| \lesssim \frac{1}{\sigma\left(J_{k}^{n+1}\right)} \int_{J_{k}^{n+1}}\left|f-\mathcal{P}_{J_{k}^{n}} f\right| d \sigma \\
& \lesssim 2^{-(2 d-1) n} E_{m}\left(f, J_{k}^{n}\right)_{1} \lesssim 2^{-(2 d-1) n} \inf \left\{E(f, z): z \in L\left(J_{k}^{n}\right)\right\} .
\end{aligned}
$$

hold.

Since $\rho(z) \asymp 2^{-d n}$,

$$
\int_{J_{k}^{n+1}}\left|T_{n+1}-T_{n}\right|^{p} d \sigma \lesssim 2^{-n(p-1)} \int_{J_{k}^{n}} E(f, z)^{p} \rho(z)^{-2 d p} d \mu_{2 d}(z) .
$$

Consider the domain $L_{n}=\left\{z \in \mathbb{C}^{d} \backslash \Omega: 2^{-n-10}<\operatorname{dist}(z, \Omega)<2^{-n}\right\}, n \geq n_{0}$; then $L\left(J_{n}^{k}\right) \subset L_{n}$,

$$
\left\|T_{n+1}-T_{n}\right\|_{L^{p}(\partial \Omega)} \lesssim 2^{-n(1-1 / p)}\left(\int_{L_{n}} E(f, z)^{p} \rho(z)^{-2 d p} d \mu_{2 d}(z)\right)^{1 / p}
$$

and

$$
\sum_{n=n_{0}}^{\infty}\left\|T_{n+1}-T_{n}\right\|_{L^{p}(\partial \Omega)} \leq\left(\int_{\mathbb{C}^{d} \backslash \Omega} E(f, z)^{p} \rho(z)^{-2 d p} d \mu_{2 d}(z)\right)^{1 / p}<\infty .
$$

We proceed to construction of a continuation of the function $f$. Consider the Witney partition in the domain $\Omega_{1} \backslash \bar{\Omega}$ with $\sum \chi_{k}=1$ and $\left|\operatorname{grad} \chi_{k}(z)\right| \lesssim \rho(z)^{-1}$. Let $z_{k} \in \operatorname{supp} \chi_{k}$ and $J_{k}=Q_{\rho\left(z_{k}\right) / 100}\left(z_{k}^{*}\right)$. We define

$$
\mathbf{f}(z)=\sum_{k=1}^{\infty} \chi_{k}(z) \mathcal{P}_{J_{k}} f(z), \quad z \in \mathbb{C}^{d} \backslash \Omega
$$


Note that for an arbitrary polynomial $T(z)$,

$$
\mathbf{f}(z)=T(z)+\sum_{k=1}^{\infty} \chi_{k}(z)\left(\mathcal{P}_{J_{k}} f(z)-T(z)\right), \quad z \in \mathbb{C}^{d} \backslash \Omega
$$

and

$$
\bar{\partial} \mathbf{f}(z)=\sum_{k=1}^{\infty}\left(\mathcal{P}_{J_{k}} f(z)-T(z)\right) \bar{\partial} \chi_{k}(z), \quad z \in \mathbb{C}^{d} \backslash \Omega .
$$

Now let $T=\mathcal{P}_{J(z)} f$. Note that $J_{k} \subset J(z)$ if $\chi_{k}(z) \neq 0$; hence, by estimate (4.3), we have the relation

$$
\left|\mathcal{P}_{J_{k}} f(z)-T(z)\right|=\left|\mathcal{P}_{J_{k}}(f-T)(z)\right| \lesssim \sigma(J(z))^{-1} E(f, z) \lesssim \rho(z)^{-(2 d-1)} E(f, z) .
$$

In addition supp $\mathbf{f} \subset \Omega_{1}$ and

$$
|\bar{\partial} \mathbf{f}(z)| \lesssim E(f, z) \rho(z)^{-2 d} \in L^{p}\left(\mathbb{C}^{d} \backslash \Omega\right) .
$$

The theorem is proved.

In the proof of this theorem, polynomials were used for construction of a continuation of the function $f$. Conversely, let a pseudoanalytic continuation of the function $f$ on the space $\mathbb{C}^{d}$ be given and let $J \subset\{\xi \in \partial \Omega$ : $\left.v\left(\xi, z_{0}\right)<h / A\right\}$ for some point $z_{0} \in \partial \Omega$, where the constant $A$ is chosen according to Lemma 2.3. Consider the polynomial of degree $m$ :

$$
P_{J}(z)=\int_{v\left(\xi, z_{0}\right)>2 h} \bar{\partial} \mathbf{f}(\xi) \wedge \omega(\xi) K_{m}^{\text {loc }}\left(\xi, z, z_{0}\right),
$$

where the form $\omega(\xi)$ is defined in (2.3) and the kernel $K_{m}^{\text {loc }}$ is defined in the following way:

$$
\begin{aligned}
K(\xi, z)= & \frac{1}{\langle\partial \rho(\xi), \xi-z\rangle^{d}}=\frac{1}{\left(\left\langle\partial \rho(\xi), \xi-z_{0}\right\rangle+\left\langle\partial \rho(\xi), z_{0}-z\right\rangle\right)^{d}} \\
= & \frac{1}{\left\langle\partial \rho(\xi), \xi-z_{0}\right\rangle^{d}}\left(1+\frac{\left\langle\partial \rho(\xi), z_{0}-z\right\rangle}{\left\langle\partial \rho(\xi), \xi-z_{0}\right\rangle}\right)^{-d} \\
= & \frac{1}{\left\langle\partial \rho(\xi), \xi-z_{0}\right\rangle^{d}}\left(1+\sum_{k=1}^{m} \frac{\left\langle\partial \rho(\xi), z_{0}-z\right\rangle^{k}}{\left\langle\partial \rho(\xi), \xi-z_{0}\right\rangle^{k}}\right. \\
& \left.\quad+O\left(\frac{\left|\left\langle\partial \rho(\xi), z_{0}-z\right\rangle\right|}{\left|\left\langle\partial \rho(\xi), \xi-z_{0}\right\rangle\right|}\right)^{m+1}\right) \\
= & K_{m}^{\text {loc }}\left(\xi, z, z_{0}\right)+O\left(\frac{\left|\left\langle\partial \rho(\xi), z_{0}-z\right\rangle\right|^{d+1}}{\left|\left\langle\partial \rho(\xi), \xi-z_{0}\right\rangle\right|^{m+d+1}}\right) .
\end{aligned}
$$

Note that, similarly to $(2.5)$, for $\left|v\left(\xi, z_{0}\right)\right|>A h$ and $\left|v\left(z, z_{0}\right)\right|<h$, we have the inequality

$$
\left|K(\xi, z)-K_{m}^{\text {loc }}\left(\xi, z, z_{0}\right)\right| \lesssim \frac{v\left(z, z_{0}\right)^{\frac{m+1}{2}}}{v(\xi, z)^{d+\frac{m+1}{2}}} .
$$

The polynomial $P_{J}$ can be used instead of the polynomial of best approximation. In this case, estimate (5.6) implies that

$$
\left|f(z)-P_{J}(z)\right| \lesssim \int_{v\left(\xi, z_{0}\right)<2 h} \frac{|\bar{\partial} \mathbf{f}(z)| d \mu_{2 d}(z)}{|\langle\partial \rho(\xi), \xi-z\rangle|^{d}}+\int_{v\left(\xi, z_{0}\right)>2 h}|\bar{\partial} \mathbf{f}(z)| \frac{h^{\frac{m+1}{2}}}{|\langle\partial \rho(\xi), \xi-z\rangle|^{d+\frac{m+1}{2}}} d \mu_{2 d}(z)
$$

for $z \in J$.

\subsection{Continuation by global approximations.}

Let a function $f \in H^{1}(\Omega)$ be approximated in $L^{1}(\partial \Omega)$ by a sequence of polynomials $P_{1}(z), P_{2}(z), \ldots$ of degree $1,2, \ldots$, correspondingly. We set

$$
\lambda(z)=\rho(z)^{-1}\left|P_{2^{n+1}}(z)-P_{2^{n}}(z)\right|, \quad 2^{-n}<\rho(z)<2^{-n+1} .
$$

Theorem 5.2. Assume that $\lambda \in L^{p}\left(\mathbb{C}^{d} \backslash \Omega\right)$ for some $p \geq 1$. Then $f \in L^{p}(\partial \Omega)$ and there exists a pseudoanalytic continuation $\mathbf{f}$ of the function $f$ such that

$$
|\bar{\partial} \mathbf{f}(z)| \lesssim \lambda(z), \quad z \in \mathbb{C}^{d} \backslash \Omega .
$$


Proof. Let a function $\chi \in C^{\infty}(0, \infty)$ be such that $\chi(t)=1$ for $t \leq 1$ and $\chi(t)=0$ for $t \geq 2$. We define the continuation function $\mathbf{f}$ by the formula

$$
\mathbf{f}(z)=P_{2^{n}}(z)+\chi\left(2^{n} \rho(z)\right)\left(P_{2^{n+1}}(z)-P_{2^{n}}(z)\right), \quad 2^{-n}<\rho(z)<2^{-n+1} .
$$

It is clear that the function $\mathbf{f}$ is continuously differentiable in $\mathbb{C}^{d} \backslash \bar{\Omega}$ and that $|\bar{\partial} \mathbf{f}(z)| \lesssim \lambda(z)$. We introduce a function $F_{N}(z)$ such that $F_{N}(z)=\mathbf{f}(z)$ for $\rho(z)>2^{-N}$ and $F_{N}(z)=P_{2^{N+1}}(z)$ for $\rho(z)<2^{-N}$. Then the function $F_{N}$ is infinitely differentiable everywhere, analytic in the domain $\Omega_{2^{-N}}$, and $\left|F_{N}(z)\right| \lesssim \lambda(z)$ outside this domain. Similarly to the previous theorem, we obtain the equality

$$
P_{2^{n+1}}(z)=F_{N}(z)=\frac{1}{(2 \pi i)^{d}} \int_{\mathbb{C}^{d} \backslash \Omega} \frac{\bar{\partial} F_{N}(\xi) \wedge\left(\partial \rho(\xi) \wedge(\bar{\partial} \partial \rho(\xi))^{d-1}\right.}{\langle\partial \rho(\xi), \xi-z\rangle^{d}}, z \in \Omega .
$$

We can pass to the limit in this formula by the dominated convergence theorem; hence, the continuation is constructed.

In applications (see Sec. 7), $P_{n}$ are polynomials of best approximation. Conversely, if an approximation with estimate (5.8) is given, then polynomials that give almost best approximation are obtained by approximation of the kernel of the Cauchy-Leray-Fantappiè formula, which is defined in Lemma 2.10.

\section{Pseudoanalytic continuation of functions from the Besov Classes}

Let $\mathbf{f}$ be a pseudoanalytic continuation of a function $f$ and let $1 \leq p \leq \infty$. We consider the following characteristic of the function $\mathbf{f}$ :

$$
S_{p}(\mathbf{f}, r)=\left(\int_{\partial \Omega_{r}}|\bar{\partial} \mathbf{f}(z)|^{p} d \sigma_{r}(z)\right)^{1 / p}, \quad r>0 .
$$

This characteristic turns out to be directly connected with the modulus of smoothness of the function $f$.

Theorem 6.1. Assume that $1 \leq p, q \leq \infty, s>0 m$ and $f \in H^{1}(\Omega)$. Then $f \in A_{p q}^{s}(\Omega)$ if and only if there exists a pseudoanalytic continuation $\mathbf{f}$ of the function $f$ such that

$$
\int_{0}^{1}\left(\frac{S_{p}(\mathbf{f}, r)}{r^{s-1}}\right)^{q} \frac{d r}{r}<\infty
$$

for $q<\infty$. For $q=\infty$,

$$
S_{p}(\mathbf{f}, r) \lesssim r^{s-1} .
$$

Proof. Let $f \in A_{p}^{s}(\Omega)$, we use the construction from Theorem 5.1 and construct a continuation of the function $f$ such that

$$
|\bar{\partial} \mathbf{f}(z)| \lesssim E(f, J(z))_{1} \rho(z)^{-2 d}
$$

Taking the estimate $E(f, J(z))_{1} \leq \sigma(J(z))^{1-\frac{1}{p}} E_{m}(f, J(z))_{p}$, into account this inequality implies that

$$
S_{p}(\mathbf{f}, r)^{p} \lesssim r^{-(2 d-1)-p} \int_{\partial \Omega_{r}} E_{m}(f, J(z))_{p}^{p} d \sigma_{r}(z)
$$

We estimate the integral in the right-hand side. The sets $J(z)$ form a covering of the boundary $\partial \Omega$, and we can choose a finite collection of sets $J_{k}=\left\{\xi \in \partial \Omega: \operatorname{pr}_{z_{k}^{*}}(\xi) \in Q_{r}\left(z_{k}^{*}\right)\right\}$ such that for every point $z \in \partial \Omega_{r}, J(z) \subset J_{k}$ for some $k$, and every point is covered by not more than $5^{2 d}$ intervals. Therefore,

$$
\int_{\partial \Omega_{r}} E_{m}(f, J(z))_{p}^{p} d \sigma_{r}(z) \lesssim r^{2 d-1} \sum_{k=1}^{N} E_{m}\left(f, J_{k}\right)_{p}^{p} \lesssim r^{2 d-1} \omega_{m}(f, 10 r)_{p}^{p},
$$

hence, $S_{p}(\mathbf{f}, r) \lesssim \omega_{m}(f, 10 r)_{p} / r$. Condition (6.2) follows from the definition of the Besov space.

Conversely, let a continuation of the function $f$ be given for which estimate (6.2) holds. Without loss of generality, we may assume that supp $\mathbf{f} \subset \Omega_{2}$. Since an arbitrary cube $\widetilde{Q}_{t}$ is contained in the Hörmander ellipsoid 
$\left\{\xi \in \partial \Omega: v\left(\xi, z_{0}\right)<C t\right\}$, where $z_{0} \in \partial \Omega$ and $C>0$ is some constant depending only on the domain $\Omega$, inequality (5.7) implies that the modulus of continuity is estimated in the following way:

$$
\omega_{m}(f, c \delta)_{p} \lesssim\|g\|_{L^{p}(\partial \Omega)}+\|h\|_{L^{p}(\partial \Omega)}
$$

for some constant $c>0$, where

$$
g(z)=\int_{v(\xi, z)<2 \delta} \frac{|\bar{\partial} \mathbf{f}(\xi)|}{|\langle\partial \rho(\xi), \xi-z\rangle|^{d}} d \mu_{2 d}(\xi), \quad z \in \partial \Omega,
$$

and

$$
h(z)=\int_{v(\xi, z)>\delta} \frac{|\bar{\partial} \mathbf{f}(\xi)| \delta^{\frac{m+1}{2}}}{|\langle\partial \rho(\xi), \xi-z\rangle|^{d+\frac{m+1}{2}}} d \mu_{2 d}(\xi), \quad z \in \partial \Omega .
$$

Consider the following functions:

$$
g_{r}(z)=\int_{\substack{\xi \in \partial \Omega_{r}, v(\xi, z)<2 \delta}} \frac{|\bar{\partial} \mathbf{f}(\xi)|}{|\langle\partial \rho(\xi), \xi-z\rangle|^{d}} d \sigma_{r}(\xi), \quad z \in \partial \Omega,
$$

and

$$
h_{r}(z)=\int_{\substack{\xi \in \partial \Omega_{r}, v(\xi, z)>\delta}} \frac{|\bar{\partial} \mathbf{f}(\xi)| \delta^{\frac{m+1}{2}}}{|\langle\partial \rho(\xi), \xi-z\rangle|^{d+\frac{m+1}{2}}} d \sigma_{r}(\xi), \quad z \in \partial \Omega
$$

Then

$$
\omega_{m}(f, \delta)_{p} \lesssim \int_{0}^{2 \delta}\left\|g_{r}\right\|_{L^{p}(\partial \Omega)} d r+\int_{0}^{1}\left\|h_{r}\right\|_{L^{p}(\partial \Omega)} d r
$$

We show that

$$
\begin{aligned}
\left\|g_{r}\right\|_{L^{p}(\partial \Omega)} & \lesssim S_{p}(\mathbf{f}, r) \log \frac{2 \delta}{r}, & & 0<r<2 \delta, \\
\left\|h_{r}\right\|_{L^{p}(\partial \Omega)} & \lesssim S_{p}(\mathbf{f}, r), & & 0<r<\delta,
\end{aligned}
$$

and

$$
\left\|h_{r}\right\|_{L^{p}(\partial \Omega)} \lesssim S_{p}(\mathbf{f}, r) \frac{\delta^{\frac{m+1}{2}}}{r^{\frac{m+1}{2}}}, \quad r>\delta .
$$

It is sufficient to verify these estimates for $p=1$ and $p=\infty$; for $1<p<\infty$, they follow from the Riesz-Thorin interpolation theorem. By estimates of Lemma 2.7,

$$
\int_{\partial \Omega}\left|g_{r}(z)\right| d \sigma(z)=\int_{\partial \Omega_{r}}|\bar{\partial} \mathbf{f}(\xi)| d \sigma(\xi) \int_{\substack{z \in \partial \Omega, v(\xi, z)<2 \delta}} \frac{d \sigma(z)}{|\langle\partial \rho(\xi), \xi-z\rangle|^{d}} \lesssim S_{1}(\mathbf{f}, r) \log \frac{\delta}{r} .
$$

for $p=1$. For $p=\infty$,

$$
\left|g_{r}(z)\right| \lesssim S_{\infty}(\mathbf{f}, r) \int_{\substack{\xi \in \partial \Omega_{r}, v(\xi, z)<2 \delta}} \frac{d \sigma(\xi)}{|\langle\partial \rho(\xi), \xi-z\rangle|^{n}} \lesssim S_{\infty}(\mathbf{f}, r) \log \frac{\delta}{r},
$$

which proves the first estimate. Estimates for the function $h_{r}$ are obtained in a similar way by estimates of Lemma 2.8.

Therefore, we have shown that

$$
\omega_{m}(f, c \delta)_{p} \lesssim \int_{0}^{2 \delta} S_{p}(\mathbf{f}, r) \frac{\delta^{\varepsilon}}{r^{\varepsilon}} d r+\delta^{\frac{m+1}{2}} \int_{\delta}^{1} S_{p}(\mathbf{f}, r) \frac{d r}{r^{\frac{m+1}{2}}}
$$


for any $\varepsilon>0$, and the condition

$$
\left(\int_{0}^{1} \omega_{m}(f, \delta)_{p}^{q} \delta^{-1-s q} d \delta\right)^{1 / q}<\infty
$$

for $1 \leq q<\infty$ follows from the Hardy inequality. In fact, we estimate the first summand in the right-hand side of formula (6.4), set $\varepsilon=s / 2$, and define

$$
F_{1}(\delta)=\int_{0}^{\delta} S_{p}(\mathbf{f}, r) \frac{d r}{r^{\varepsilon}} .
$$

Then

$$
\begin{aligned}
\int_{0}^{1}\left(\delta^{\varepsilon} F_{1}(\delta)\right)^{q} \delta^{-1-s q} d \delta & \lesssim \int_{0}^{1} r^{-1-s q+\varepsilon q}\left(S_{p}(\mathbf{f}, r) r^{-\varepsilon} r\right)^{q} d r \\
& =\int_{0}^{1}\left(\frac{S_{p}(\mathbf{f}, r)}{r^{s-1}}\right)^{q} \frac{d r}{r}<\infty .
\end{aligned}
$$

The second summand in the right-hand side of formula (6.4) is estimated in a similar way assuming that $s<(m+1) / 2$ (note that we can choose the value of $m$ as large as needed since condition (1.5) does not depend on $m>s)$. Next, we define

$$
F_{2}(\delta)=\int_{0}^{\delta} S_{p}(\mathbf{f}, r) \frac{d r}{r^{\frac{m+1}{2}}},
$$

then

$$
\begin{aligned}
\int_{0}^{1}\left(\delta^{\frac{m+1}{2}} F_{2}(\delta)\right)^{q} \delta^{-1-s q} d \delta & \lesssim \int_{0}^{1} r^{-1-s q+\frac{m+1}{2} q}\left(S_{p}(\mathbf{f}, r) r^{-\frac{m+1}{2}} r\right)^{q} d r \\
& =\int_{0}^{1}\left(\frac{S_{p}(\mathbf{f}, r)}{r^{s-1}}\right)^{q} \frac{d r}{r}<\infty .
\end{aligned}
$$

For $q=\infty$, the estimate $\omega_{m}(f, \delta)_{p} \lesssim \delta^{s}$ is also easily obtained from inequality (6.4) and the fact that $S_{p}(\mathbf{f}, r) \lesssim r^{s-1}$. Therefore, the proof of the theorem is completed.

\section{Constructive characteristic of the Besov Classes}

Let $P_{n}$ be a sequence of polynomials of best approximation. By Theorem 5.2, we can construct a pseudoanalytic continuation $\mathbf{f}$ of the function $f$ such that

$$
|\bar{\partial} \mathbf{f}(z)| \lesssim \rho(z)^{-1}\left|P_{2^{n+1}}(z)-P_{2^{n}}(z)\right|, \quad 2^{-n} \leq \rho(z) \leq 2^{-n+1} .
$$

Assume that $0 \in \Omega$. Any point $z \in \mathbb{C}^{d}$ can be represented in the form $z=u v$, where $u=r e^{i \varphi} \in \mathbb{C}$ and

$$
v \in C=\left\{\left(v_{1}, \ldots, v_{d}\right) \in \mathbb{C}^{d}: v_{1} \in[0,1], v_{1}^{2}+\left|v_{2}\right|^{2}+\cdots+\left|v_{d}\right|^{2}=1\right\} .
$$

We write in these coordinates the boundary of the domain $\Omega_{\delta}, 0 \leq \delta \leq 1$. Note that any vector $v \in C$ generates the complex plane $T_{v}=\{u v: u \in \mathbb{C}\}$ such that the intersection of the domain $\Omega_{\delta}$ with this plane is a convex domain with the boundary $\Omega_{v, \delta}$, and the boundary of the domain depends on parameters $v$ and $\delta$ continuously. Therefore,

$$
\partial \Omega_{\delta}=\left\{r_{\delta} e^{i \varphi} v \in \mathbb{C}^{d}, r_{\delta}=r(\delta, \varphi, v)\right\}=\left\{r(\delta, \varphi, v) e^{i \varphi} v, \varphi \in[0,2 \pi], v \in C\right\},
$$

and parameters $(\varphi, v) \in[0,2 \pi] \times C$ define on $\partial \Omega_{\delta}$ smooth coordinates. Since $r_{\delta}$ also depends on $\delta$ continuously, the relation $d \sigma_{\delta} \asymp|d u| d \mu_{2 d-2}\left(v_{2}, \ldots, v_{d}\right)$ holds uniformly in $\delta \in[0,1]$. The last relation obviously holds in the case of the sphere $\mathbb{S}_{2 d-1}=\left\{z \in \mathbb{C}^{d}:|z|=1\right\}$, but a strictly convex domain is diffeomorphic to the sphere by the 
mapping $z \rightarrow \frac{z}{|z|}$; hence, the statement holds in the general case. The uniformity in $\delta \in[0,1]$ follows from the smoothness of the function $r(\delta, \varphi, v)$.

Fix a vector $v \in C$. As before, it is associated with the collection of domains $\Omega_{v, \delta}=\left\{u v \in \Omega_{\delta}: u \in \mathbb{C}\right\}$. We identify the plane $T_{v}$ with the space $\mathbb{C}$ and consider domains of the form

$$
\widetilde{\Omega}_{v, \delta}=\left\{u \in \mathbb{C}: u v \in \Omega_{v, \delta}\right\} .
$$

Consider a conformal mapping $\psi_{v}$ of the domain $\mathbb{C} \backslash \widetilde{\Omega}_{v, 0}$ on the complement of the disk $\{u \in \mathbb{C}:|u|>1\}$ normed by the condition $\psi_{v}^{\prime}(\infty)>0$. By virtue of the smoothness of the boundary, the mapping $\psi_{v}$ is continued to a conformal mapping of the closed domains and continuously depends on $v \in C$, which implies that $\left|\psi_{v}(u)\right| \asymp$ $1+\operatorname{dist}\left(u, \widetilde{\Omega}_{v, 0}\right) \asymp 1+\rho(u v)$.

Consider the function

$$
\Phi_{v}(u)=\frac{P_{2^{n+1}}(u v)-P_{2^{n}}(u v)}{\psi_{v}(u)^{2^{n+2}}},
$$

which is analytic in $\mathbb{C} \backslash \widetilde{\Omega}_{v, 0}$ and such that $|u| \Phi_{v}(u) \rightarrow 0, u \rightarrow \infty$; hence,

$$
\left\|\Phi_{v}\right\|_{H^{p}\left(\mathbb{C} \backslash \widetilde{\Omega}_{v, 0}\right)} \asymp \sup _{0<\delta<\infty}\left(\int_{\partial \widetilde{\Omega}_{v, \delta}}\left|\Phi_{v}(u)\right|^{p}|d u|\right)^{1 / p} \lesssim c\left(\Omega_{v, 0}\right)\left(\int_{\partial \tilde{\Omega}_{v, 0}}\left|\Phi_{v}(u)\right|^{p}|d u|\right)^{1 / p} .
$$

The constant $c\left(\Omega_{v, 0}\right)>0$ is determined by the geometry of the domain $\Omega_{v, 0}$ and depends on $v$ continuously; hence, $c\left(\Omega_{v, 0}\right)<c<\infty$.

Note, that $\left|\psi_{v}(u)\right|^{2^{n+2}} \asymp\left(1+2^{-n}\right)^{2^{n+2}} \asymp 1$ for $u \in \Omega_{v, 2^{-n}} \backslash \Omega_{v, 2^{-n-1}}$ and $\left|\psi_{v}(u)\right|=1$ for $u \in \partial \Omega_{v, 0}$, which implies that

$$
\begin{aligned}
\left(\int_{\partial \widetilde{\Omega}_{v, \delta}}\left|P_{2^{n+1}}(u v)-P_{2^{n}}(u v)\right|^{p}|d u|\right)^{1 / p} & \lesssim\left(\int_{\partial \widetilde{\Omega}_{v, \delta}}\left|\Phi_{v}(u)\right|^{p}|d u|\right)^{1 / p} \lesssim\left(\int_{\partial \widetilde{\Omega}_{v, 0}}\left|\Phi_{v}(u)\right|^{p}|d u|\right)^{1 / p} \\
& =\left(\int_{\partial \widetilde{\Omega}_{v, 0}}\left|P_{2^{n+1}}(u v)-P_{2^{n}}(u v)\right|^{p}|d u|\right)^{1 / p}, \quad 2^{-n} \leq \delta<2^{-n+1},
\end{aligned}
$$

where all the estimates are uniform. This fact combined with the relation $d \sigma_{\delta} \asymp|d u| d \mu_{2 d-2}$ gives us an estimate of the integrals over the boundaries of the domains $\partial \Omega_{\delta}$ :

$$
\left(\int_{\partial \Omega_{\delta}}\left|P_{2^{n+1}}(z)-P_{2^{n}}(z)\right|^{p} d \sigma_{\delta}(z)\right)^{1 / p} \lesssim\left(\int_{\partial \Omega}\left|P_{2^{n+1}}(z)-P_{2^{n}}(z)\right|^{p} d \sigma(z)\right)^{1 / p}, \quad 2^{-n} \leq \delta<2^{-n+1} .
$$

Therefore, taking into account property (7.1) of the continuation function $\mathbf{f}$, we obtain the estimate

$$
S_{p}(\mathbf{f}, \delta) \lesssim 2^{n} E_{2^{n}}(f)_{p}, \quad 2^{-n}<\delta \leq 2^{-n+1} .
$$

Theorem 7.1. Assume that $1 \leq p, q \leq \infty, s>0$, and $f \in H^{p}(\partial \Omega)$. Then $f \in A_{p q}^{s}(\Omega)$ if and only if

$$
\left(\sum_{n=1}^{\infty} \frac{1}{n}\left(n^{s} E_{n}(f)_{p}\right)^{q}\right)^{1 / q}<\infty
$$

for $q<\infty ;$ for $q=\infty$,

$$
E_{n}(f)_{p} \lesssim n^{-s}, \quad n=1, \ldots, \infty .
$$

Proof. Note that $E_{n}(f)_{p}$ monotonically decreases with growth of $n$, hence, condition (7.3) is equivalent to the condition

$$
\left(\sum_{n=1}^{\infty} 2^{n s q} E_{2^{n}}(f)_{p}^{q}\right)^{1 / q}<\infty
$$


Assume that condition (7.3) holds. Then, using the construction from Theorem 5.2, we can construct a pseudoanalytic continuation $\mathbf{f}$ of the function $f$ such that

$$
S_{p}(\mathbf{f}, r) \lesssim 2^{n} E_{2^{n}}(f)_{p}, \quad 2^{-n} \leq r \leq 2^{-n+1},
$$

and

$$
\left(\int_{0}^{1}\left(\frac{S_{p}(\mathbf{f}, r)}{r^{s-1}}\right)^{q} \frac{d r}{r}\right)^{1 / q} \lesssim\left(\sum_{n=1}^{\infty} 2^{n s} E_{2^{n}}(f)_{p}^{q}\right)^{1 / q}<\infty
$$

which implies that $f \in A_{p q}^{s}(\Omega)$ by Theorem 6.1 .

Conversely, assume that $f \in A_{p q}^{s}(\Omega)$. Then there exists a pseudoanalytic continuation of the function $f$ satisfying estimate (6.2). We prove a more accurate estimate of the best approximations:

$$
E_{n}(f)_{p} \lesssim \int_{0}^{1 / n} S_{p}(\mathbf{f}, r) \frac{d r}{(n r)^{\varepsilon}} d r+\int_{1 / n}^{\infty} S_{p}(\mathbf{f}, r) \frac{d r}{(n r)^{\alpha}}
$$

where the value of parameter $\alpha$ is as large as needed and the value of $\varepsilon>0$ is small. We construct polynomials approximating the function $f$. For this purpose, we use the estimates of the Cauchy-Leray-Fantappiè kernel from Lemma 2.10 and put

$$
P_{n}(z)=\int_{\mathbb{C}^{d} \backslash \Omega} \bar{\partial} \mathbf{f} \wedge \omega(\xi) K_{n}^{\mathrm{glob}}(\xi, z)
$$

Then

$$
\begin{aligned}
\left|f(z)-P_{n}(z)\right| & \lesssim \int_{\mathbb{C}^{d} \backslash \Omega}|\bar{\partial} \mathbf{f}|\left|\frac{1}{\langle\partial \rho(\xi), \xi-z\rangle^{d}}-K_{n}^{\text {glob }}(\xi, z)\right| d \mu_{2 d}(\xi) \\
& \leq U(z)+V(z)+W_{1}(z)+W_{2}(z)
\end{aligned}
$$

where $\mu_{2 d}$ is the Lebesgue measure in $\mathbb{C}^{d}$, and

$$
\begin{aligned}
U(z) & =\int_{v(\xi, z)<1 / n} \frac{|\bar{\partial} \mathbf{f}(\xi)|}{|\langle\partial \rho(\xi), \xi-z\rangle|^{d}} d \mu_{2 d}(\xi), \\
V(z) & =n^{d} \int_{v(\xi, z)<1 / n}|\bar{\partial} \mathbf{f}(\xi)| d \mu_{2 d}(\xi), \\
W_{1}(z) & =\frac{1}{n^{\alpha}} \int_{v(\xi, z)<1 / n,} \frac{|\bar{\partial} \mathbf{f}(\xi)|}{|\langle\partial \rho(\xi), \xi-z\rangle|^{d+\alpha}} d \mu_{2 d}(\xi), \\
W_{2}(z) & =\frac{1}{n^{\alpha}} \int_{\rho(z)>1 / n} \frac{|\bar{\partial} \mathbf{f}(\xi)|}{|\langle\partial \rho(\xi), \xi-z\rangle|^{d+\alpha}} d \mu_{2 d}(\xi),
\end{aligned}
$$

where $\alpha$ is some fixed value that is more than the exponent $s$.

Note that $V(z) \leq U(z)$. Next,

$$
U(z) \lesssim \int_{0}^{1 / n} d r \int_{\partial \Omega_{r}} \frac{|\bar{\partial} \mathbf{f}(\xi)| d \sigma_{r}(\xi)}{|\langle\partial \rho(\xi), \xi-z\rangle|^{d}}=\int_{0}^{1 / n} g_{r}(z) d r .
$$

It is easily shown that $\left\|g_{r}\right\|_{L^{p}(\partial \Omega)} \lesssim \int_{0}^{1 / n} S_{p}(\mathbf{f}, r) \log \frac{2}{n r} d r$, which implies, by the Minkowski integral inequality, that

$$
\|U\|_{p} \lesssim \int_{0}^{1 / n} S_{p}(\mathbf{f}, r) \log \frac{2}{n r} d r
$$


Similarly, we obtain the estimates

$$
\left\|W_{1}\right\|_{p} \lesssim \int_{0}^{1 / n} S_{p}(\mathbf{f}, r) d r \quad \text { and } \quad\left\|W_{2}\right\|_{p} \lesssim \int_{1 / n}^{\infty} S_{p}(\mathbf{f}, r) \frac{d r}{(n r)^{\alpha}}
$$

which completes the proof of inequality (7.5).

The final estimate (7.3) for $1 \leq q<\infty$ follows from the Hardy inequality. Actually, we put $F(t)=\int_{0}^{t} S_{p}(\mathbf{f}, r) \frac{d r}{r^{\varepsilon}}$, and let $\varepsilon<s$; hence, due to the monotonicity of the function $F$,

$$
\begin{aligned}
\sum_{n=1}^{\infty} n^{s q-1}\left(\int_{0}^{1 / n} S_{p}(\mathbf{f}, r) \frac{d r}{(n r)^{\varepsilon}}\right)^{q} & \lesssim \int_{0}^{\infty} t^{(\varepsilon-s) q-1} F(t)^{q} d t \\
& \lesssim \int_{0}^{\infty} r^{(\varepsilon-s) q-1}\left(S_{p}(\mathbf{f}, r) r^{1-\varepsilon}\right)^{q} d r=\int_{0}^{\infty} S_{p}(\mathbf{f}, r)^{q} r^{-q(s-1)-1} d r<\infty
\end{aligned}
$$

Let

$$
G(t)=\int_{t}^{\infty} S_{p}(\mathbf{f}, r) \frac{d r}{r^{\alpha}}
$$

In this case, $\alpha>s$; hence,

$$
\begin{aligned}
\sum_{n=1}^{\infty} n^{s q-1}\left(\int_{1 / n}^{\infty} S_{p}(\mathbf{f}, r) \frac{d r}{(n r)^{\alpha}}\right)^{q} & \lesssim \int_{0}^{\infty} t^{(\alpha-s) q-1} G(t)^{q} d t \\
& \lesssim \int_{0}^{\infty} r^{(\alpha-s) q-1}\left(S_{p}(\mathbf{f}, r) r^{1-\alpha}\right)^{q} d r=\int_{0}^{\infty} S_{p}(\mathbf{f}, r)^{q} r^{-q(s-1)-1} d r<\infty
\end{aligned}
$$

Therefore, it follows from estimates (7.5)-(7.7) that

$$
\sum_{n=1}^{\infty} \frac{1}{n}\left(n^{s} E_{n}(f)_{p}\right)^{q} \lesssim \int_{0}^{\infty}\left(\frac{S_{p}(\mathbf{f}, r)}{r^{s-1}}\right)^{q} \frac{d r}{r}<\infty
$$

For $q=\infty$, we have the inequality $E_{n}(f)_{p} \lesssim n^{-s}$, and the theorem is proved.

\section{Boundedness of the Cauchy-Leray-Fantappiè operator in the Besov spaces}

Theorem 6.1 has many interesting applications, e.g., the continuity of the Cauchy-Leray-Fantappiè operator in some Besov spaces can be deduced from its proof.

Theorem 8.1. Assume that $1<p<\infty, 1 \leq q \leq \infty$, and $0<s<1$. Then the operator $K_{d}$ continuously maps the space $B_{p q}^{s}(\partial \Omega)$ onto the space $A_{p q}^{s}(\Omega)$, and

$$
\left\|K_{d} f\right\|_{A_{p q}^{s}(\Omega)} \leq c(\Omega, p, q, s)\|f\|_{B_{p q}^{s}(\partial \Omega)} .
$$

Proof. Recall that $\|f\|_{B_{p q}^{s}(\partial \Omega)}=\|f\|_{L^{p}(\partial \Omega)}+\widetilde{c}_{p q}(f)$, where $\widetilde{c}_{p q}(f)$ is defined in Theorem 3.4. The operator $K_{d}$ is bounded on $L^{p}(\partial \Omega)$ due to [7]; hence, $K_{d} f \in L^{p}(\partial \Omega)$. Now let $f \in B_{p q}^{s}(\partial \Omega)$. By the constructions of Sec. 3, using only local approximations by constants, we construct a continuation $\mathbf{f}$ of the function $f$ such that $|\bar{\partial} \mathbf{f}| \leq E(f, z) \rho(z)^{-2 d}$. Therefore, similarly to Theorem $6.1, S_{p}(\mathbf{f}, r) \lesssim \omega_{m}(f, 10 r) / r$. In addition, similarly to 
formula $(2.2)$,

$$
\begin{aligned}
& f(z)=\lim _{r \rightarrow 0+} \frac{1}{(2 \pi i)^{d}} \int_{\partial \Omega_{r}} \frac{\mathbf{f}(\xi) \partial \rho(\xi) \wedge(\bar{\partial} \partial \rho(\xi))^{d-1}}{\langle\partial \rho(\xi), \xi-z\rangle^{d}} \\
& =\lim _{r \rightarrow 0+} \frac{1}{(2 \pi i)^{d}} \int_{\mathbb{C}^{d} \backslash \Omega_{r}} \frac{\bar{\partial} \mathbf{f}(\xi) \wedge \partial \rho(\xi) \wedge(\bar{\partial} \partial \rho(\xi))^{d-1}}{\langle\partial \rho(\xi), \xi-z\rangle^{d}} \\
& =\frac{1}{(2 \pi i)^{d}} \int_{\mathbb{C}^{d} \backslash \Omega} \frac{\bar{\partial} \mathbf{f}(\xi) \wedge \partial \rho(\xi) \wedge(\bar{\partial} \partial \rho(\xi))^{d-1}}{\langle\partial \rho(\xi), \xi-z\rangle^{d}} .
\end{aligned}
$$

As in Theorem 6.1, repeating the reasoning of estimate (5.7), from the previous formula we obtain the inequality

$$
\omega_{m}\left(K_{d} f, \delta\right)_{p} \lesssim \int_{0}^{2 \delta} S_{p}(\mathbf{f}, r) \frac{\delta^{\varepsilon}}{r^{\varepsilon}} d r+\delta^{\frac{m+1}{2}} \int_{\delta}^{\infty} S_{p}(\mathbf{f}, r) \frac{d r}{r^{\frac{m+1}{2}}} .
$$

This inequality implies that

$$
\begin{aligned}
\widetilde{c}_{p q}\left(K_{d} f\right)^{q} & =\int_{0}^{1}\left(\frac{\omega_{m}\left(K_{d} f, \delta\right)_{p}}{\delta^{s}}\right)^{q} \frac{d \delta}{\delta} \lesssim \int_{0}^{1}\left(\frac{S_{p}(\mathbf{f}, r)_{p}}{r^{s-1}}\right)^{q} \frac{d r}{r} \\
& \lesssim \int_{0}^{1}\left(\frac{\omega_{m}(f, r)_{p}}{r^{s}}\right)^{q} \frac{d r}{r} \lesssim \widetilde{c}_{p q}(f)^{q} .
\end{aligned}
$$

The last inequality together with the continuity of the operator $K_{d}$ on $L^{p}(\partial \Omega)$ proves the theorem.

Note that due to [6], the operator $K_{d}$ is also bounded in the Hölder space $\Lambda^{s}(\partial \Omega)=B_{\infty}^{s}(\partial \Omega)$ for $0<s<1$.

\section{Conclusion}

The characteristic obtained in Theorem 7.1 is, in general, similar to the characteristic of the Besov classes obtained by E. M. Dyn'kin in [5], however, its formulation is closer to a similar statement for the periodic Besov classes on $[-\pi, \pi]$. The reason of such simplification is the smoothness of the domain, which is necessary for applying the Cauchy-Leray-Fantappiè formula. Note that in the one-dimensional case, assuming the smoothness of the boundary of the domain, we pass to condition (7.3). The final result can be formulated as a general statement.

Theorem 9.1. Assume that $\Omega \subset \mathbb{C}^{d}$ is a domain with smooth boundary which is strictly convex in terms of (1.1). Let $1 \leq p, q \leq \infty, s>0$, and $f \in H^{p}(\partial \Omega)$. Then $f \in A_{p q}^{s}(\Omega)$ if and only if

$$
\left(\sum_{n=1}^{\infty} \frac{1}{n}\left(n^{s} E_{n}(f)_{p}\right)^{q}\right)^{1 / q}<\infty, \quad 1 \leq q<\infty,
$$

and

$$
E_{n}(f)_{p} \lesssim n^{-s}, \quad q=\infty .
$$

Note also that the methods of pseudoanalytic continuation suggested in Sec. 5 are important in themselves because they enable one to study the smoothness of the boundary values of analytic functions and obtain conditions which are similar to Theorem 6.1.

Translated by K. S. Pilyugin. 


\section{REFERENCES}

1. L. A. Aizenberg and A. P. Yuzhakov, Integral Representations and Residues in Complex Analysis [in Russian], Moscow (1979).

2. Yu. A. Brudnyi, "Spaces that are definable by means of local approximations," Trudy Moskov. Mat. Ob., 24, 69-132 (1971).

3. Yu. A. Brudnyi J. P. Irodova, "Nonlinear spline approximation of functions of several variables and $B$-spaces," Algebra Analiz, 4, 45-79 (1992).

4. V. K. Dzyadyk, Introduction to the Theory of Uniform Approximation of Functions by Polynomials [in Russian], Moscow (1977).

5. E. M. Dyn'kin, "Constructive characterization of S. L. Sobolev and O. V. Besov classes," Trudy Mat. Inst. AN SSSR, 155, 41-76 (1981).

6. A. S. Rotkevich, "The Aizenberg formula in nonconvex domains and some of its applications," Zap. Nauchn. Semin. POMI, 389, 206-231 (2011).

7. A. S. Rotkevich, "The Cauchy-Leray-Fantappiè integral in linearly convex domains," Zap. Nauchn. Semin. POMI, 201, 172-188 (2012).

8. G. H. Hardy, J. E. Littlewood, and G. Pòlya, Inequalities [Russian translation], Moscow (1948).

9. N. A. Shirokov, "A direct theorem in a strictly convex domain in $\mathbb{C}^{n}, "$ Zap. Nauchn. Semin. POMI, 206, 151-173 (1913).

10. N. A. Shirokov, "Uniform polynomial approximations in convex domains in $\mathbb{C}^{n}$," Zap. Nauchn. Semin. POMI, 333, 98-112 (2006).

11. K. Adachi, Several complex variables and integral formulas, World Scientific (2007).

12. F. Beatrous Jr., "Estimates for continuations of holomorphic functions," Michigan Math. J., 32, 361-380 (1985).

13. Bloom T. et al. Polynomial interpolation and approximation in $\mathbb{C}^{d},(2011)$ arXiv preprint arXiv:1111.6418.

14. C. Fefferman and E. M. Stein, "H $H^{p}$ spaces of several variables," Acta Math., 129, no. 1, 137-193 (1972).

15. J. Leray, "Le calcul différentiel et intégral sur une variété analytique complexe. (Problème de Cauchy. III)," Bulletin de la Société mathématique de France, 87, 81-180 (1959).

16. N. Levenberg, "Approximation in $\mathbb{C}^{N}$," Surv. Appr. Theory, 2, 92-140 (2006).

17. R. M. Range, Holomorphic functions and integral representations in several complex variables, SpringerVerlag (1986).

18. W. Rudin, Function theory in the unit ball of $\mathbb{C}^{d}$, Springer-Verlag (1980).

19. M. Simon Salamon, "Hermitian geometry," in: Invitations to Geometry and Topology, Oxf. Grad. Texts Math, 7, 233-291 (2002).

20. N. A. Shirokov, "Jackson-Bernstein theorem in strictly pseudoconvex domains in $\mathbb{C}^{n}$," Constructive Approximation, 5, no. 1, 455-461 (1989).

21. E. L. Stout, " $H^{p}$-functions on strictly pseudoconvex domains," Amer. J. Math., 98, no. 3 (Autumn, 1976), $821-852$.

22. H. Triebel, Theory of function spaces. III, Birkhauser Basel 3 (2006). 